\title{
Facile Decomposition of Organophosphonates by Dual Lewis Sites on a $\mathrm{Fe}_{3} \mathrm{O}_{4}(111)$ Film
}

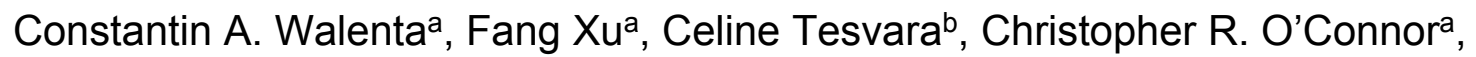
Philippe Sautet ${ }^{\mathrm{b}, \mathrm{c}}$, Cynthia M. Friend ${ }^{*}, \mathrm{a}, \mathrm{d}$

aDepartment of Chemistry \& Chemical Biology, Harvard University, Cambridge, MA 02138, USA

b Chemical and Biomolecular Engineering Department, University of California, Los Angeles, Los Angeles, CA 90095, USA

c Chemistry and Biochemistry Department, University of California, Los Angeles, Los Angeles, CA 90095, USA

dJohn A. Paulson School of Engineering and Applied Sciences, Harvard University, Cambridge, MA 02138, USA

Corresponding Author: friend@fas.harvard.edu

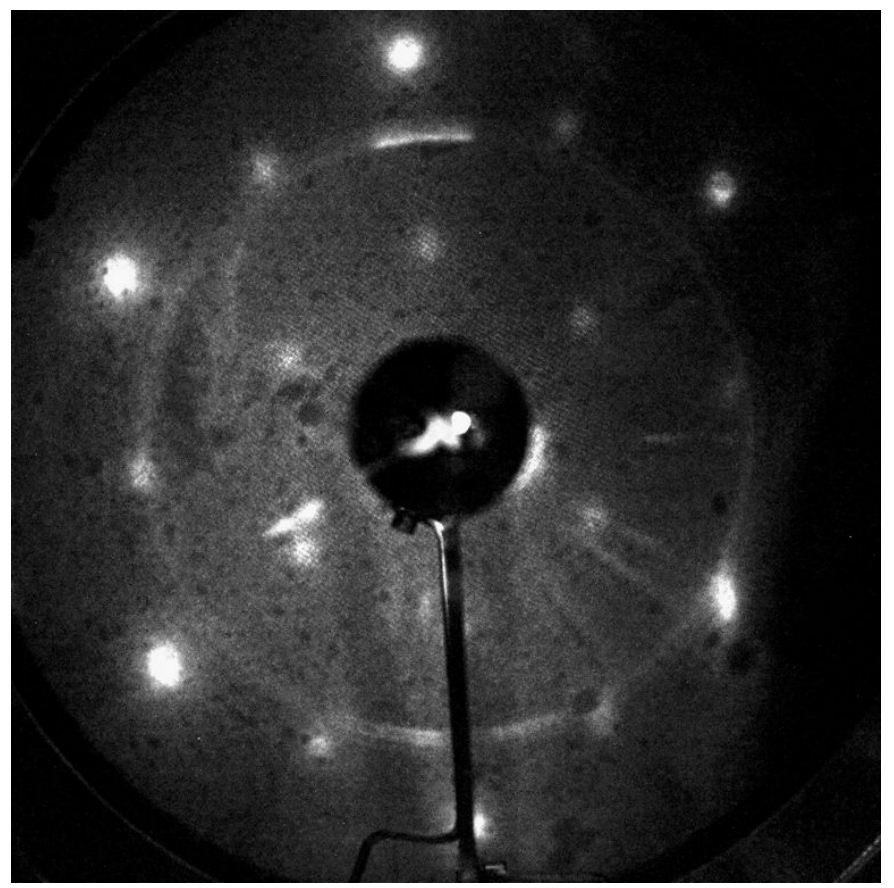

Fig. S1: Low Energy Electron Diffraction Image of the $\mathrm{Fe}_{3} \mathrm{O}_{4}(111)$ termination of the $\mathrm{Fe}_{2} \mathrm{O}_{3}(0001)$ single crystal at $81 \mathrm{eV}$. The characteristic $(2 \times 2)$ diffraction pattern with respect to the oxygen packed lattice is observed. ${ }^{1-2}$ Note, that a higher incident electron beam voltage is required in comparison to earlier work due to charging effects. ${ }^{3}$ 
Table S1: Ionization cross sections used to determine the amount gaseous products during the temperature programmed reaction experiments in this work. A quantification with regard to the initial dimethoxy methylphosphonate signal is impossible, since there is no literature value for the ionization cross section.

\begin{tabular}{c||c|c} 
Molecule & Ionization Cross Section $/ \AA^{2}$ & Reference \\
\hline \hline Dimethyl ether & 9.33 & 4 \\
\hline Formaldehyde & 4.14 & 5 \\
\hline Methanol & 4.61 & 6 \\
\hline Carbon Monoxide & 2.516 & 7 \\
\hline Carbon Dioxide & 3.521 & 7 \\
\hline Water & 2.275 & 7
\end{tabular}
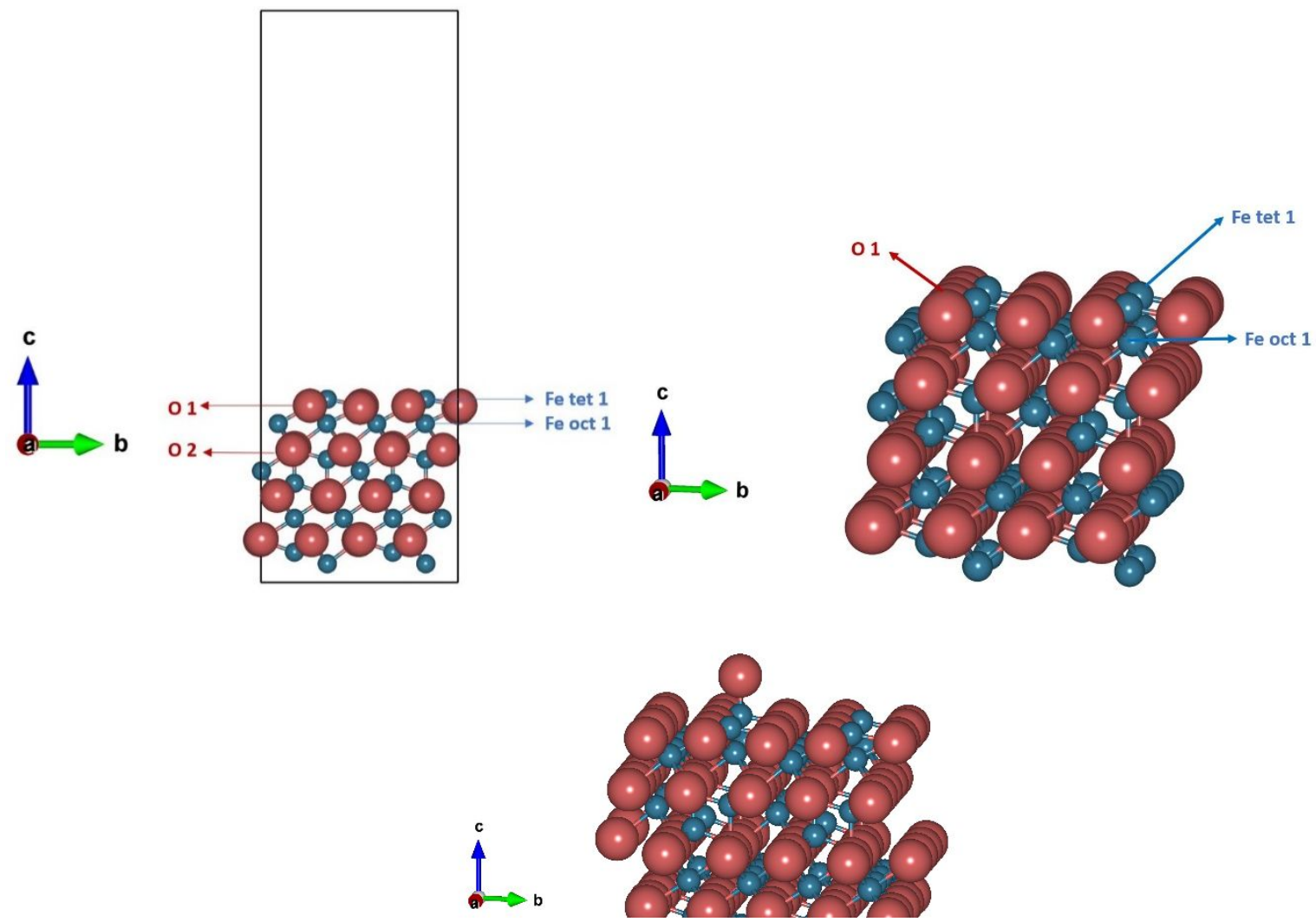

Fig S2: (Top) Side and perspective views of $\mathrm{Fe}_{3} \mathrm{O}_{4}$ slab model. (Bottom) Surface with $1 / 4 \mathrm{ML} \mathrm{O}$ coverage. Blue represents $\mathrm{Fe}$ atoms and red represents Oxygen atoms. Each supercell contains 4 surface tetrahedral Fe.

The adsorption energies are calculated using:

$$
E_{\text {adsorption }}=E_{\text {surf }+D M M P}-E_{\text {surf } \frac{1}{4} M L O}-E_{D M M P}
$$


The second most stable molecular adsorption corresponds to conformation (2) where one oxygen of DMMP methoxy binds to tetrahedral Fe with adsorption energy of -2.06 $\mathrm{eV}$ (Fig. 3). The distance of $\left(\mathrm{O}_{\text {DMMP }}\right)---\left(\mathrm{Fe}_{\text {tet }}\right)_{\text {DMMP }}$ is $2.03 \AA$. There are additional interactions of DMMP with O-adatom involving both methoxy and methyl group of DMMP with distance of closest $\mathrm{H}_{\text {methoxy }}--\mathrm{O}_{\text {adatom }}=2.44 \AA$ and closest $\mathrm{H}_{\text {methyl---O }}-\mathrm{O}_{\text {adatom }}$ $=3.33 \AA$.

Table S2: Adsorption energies of molecularly adsorbed DMMP on $\mathrm{Fe}_{3} \mathrm{O}_{4}$ (111). Table 1 lists 2 thermodynamically stable ways to adsorb DMMP on the surface alongside with its adsorption energy, starting with: (1) oxygen from the methoxy of DMMP interacting with surface Fe tetrahedral cation and (2) P of DMMP interacting directly with $\mathrm{O}$ adatom on the surface.

\begin{tabular}{|c|c|c|c|c|}
\hline \multirow[t]{2}{*}{ Index } & \multicolumn{2}{|c|}{ Molecular adsorption modes } & \multirow{2}{*}{$\begin{array}{l}\text { Adsorption } \\
\text { Energy [eV] }\end{array}$} & \multirow{2}{*}{$\begin{array}{c}\text { Adsorption } \\
\text { Energy }[\mathrm{kJ} / \mathrm{mol}]\end{array}$} \\
\hline & DMMP & Surface & & \\
\hline 1 & $\begin{array}{c}\mathrm{O} \text { of } \\
\text { methoxy }\end{array}$ & Fe tet & -2.06 & -198.76 \\
\hline 2 & $\mathrm{O}$ of $\mathrm{P}=\mathrm{O}$ & Fe tet & -2.57 & -247.97 \\
\hline
\end{tabular}

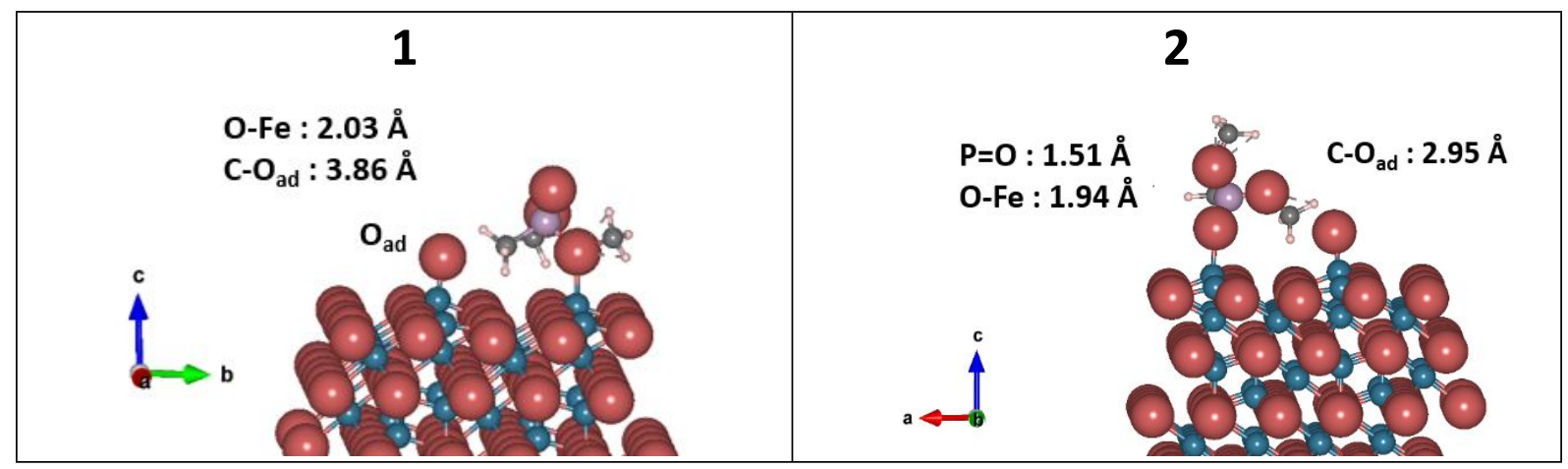

Fig. S3: Relaxed geometries of DMMP adsorbed molecularly on $\mathrm{Fe}_{3} \mathrm{O}_{4}(111)$ via various modes. The energies are given in Table S2. 


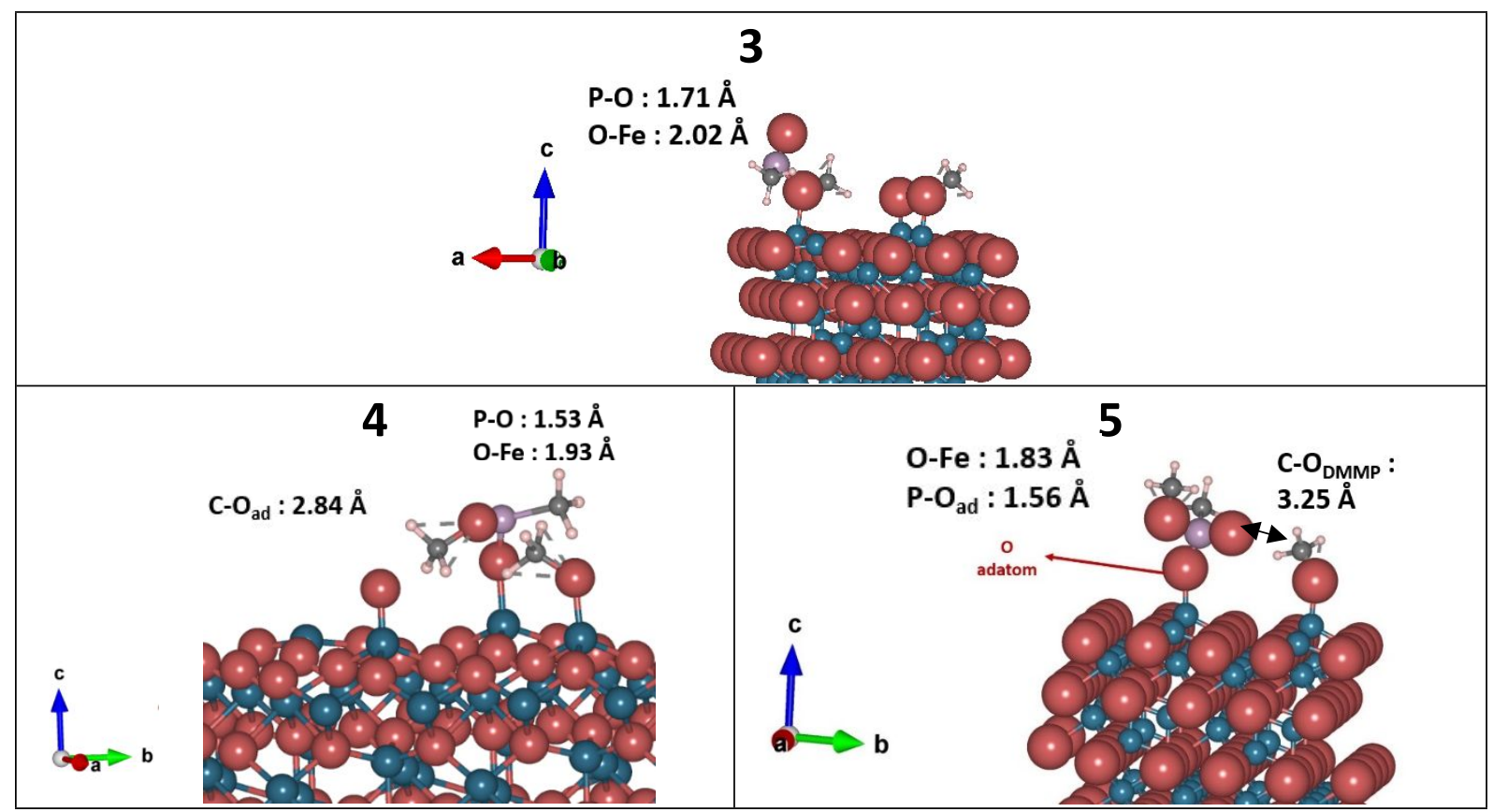

Fig. S4: Different MMP and methoxy configurations on the $\mathrm{Fe}_{3} \mathrm{O}_{4}(111)$ surface with an oxygen adatom. The respective energies are displayed in Table S3.

Table S3: The three energies of dissociative adsorption conformations along with their adsorption energy relative to gas phase DMMP, consisting for the MMP fragment of: (5) Oxygen of the methoxy on MMP interacting with surface tetrahedral Fe cation, (6) Oxygen of MMP phosphonate group interacting with surface Fe tetrahedral cation, and (7) P of MMP interacting with O-adatom on the surface.

\begin{tabular}{|l|l|l|c|c|}
\hline \multirow{2}{*}{ Index } & \multicolumn{2}{|l|}{ Dissociative adsorption modes } & Adsorption Energy & $\begin{array}{l}\text { Adsorption Energy } \\
{[\mathrm{kJ} / \mathrm{mol}]}\end{array}$ \\
\cline { 2 - 4 } & $\mathrm{MMP}$ & Surface & -0.23 & -22.19 \\
\hline 3 & $\mathrm{CH} 3 \mathrm{O}$ & Fe tet & -0.83 & -80.01 \\
\hline 4 & $\mathrm{P}=\mathrm{O}$ & Fe tet & -3.76 & -362.78 \\
\hline 5 & $\mathrm{P}$ & O-Fe tet & & \\
\hline
\end{tabular}




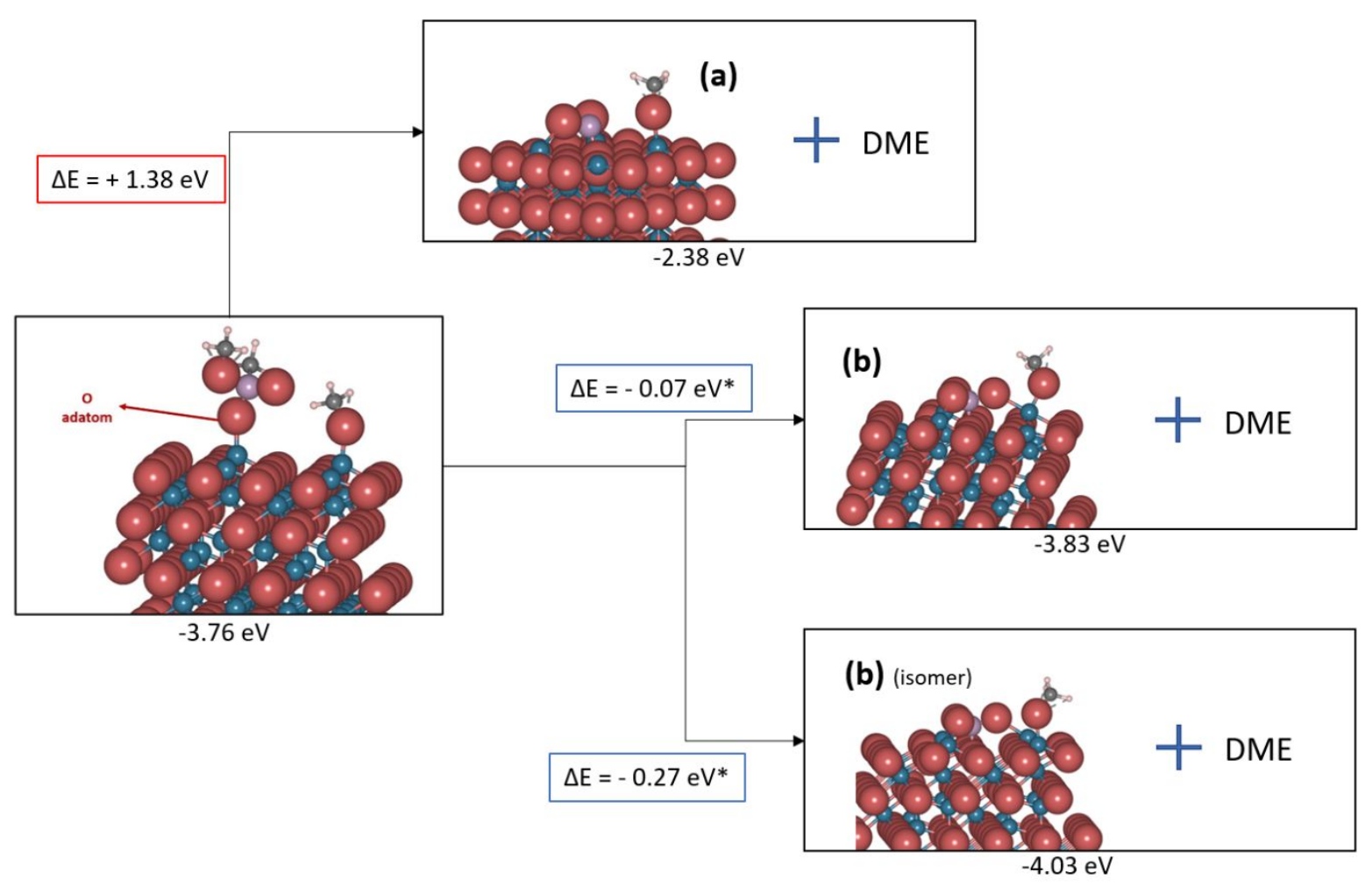

Fig. S5: Possible DME formation pathway from MMP and Methoxy on the surface. Direct production of DME from MMP is not thermodynamically possible (intermediate a). Formation of DME would only be thermodynamically possible if extra O-adatom exists to stabilize the phosphate group (intermediate b). * denotes energy difference after O-adatom stabilization. In Intermediate $b$ isomer, surface methoxy is moved towards neighboring free Fe-tetrahedral site.

The oxygen chemical potential required for this transformation is obtained through hematite reduction reaction to magnetite:

$$
3 \mathrm{Fe}_{2} \mathrm{O}_{3 \text { bulk }} \rightarrow 2 \mathrm{Fe}_{3} \mathrm{O}_{4 \text { bulk }}+\mathrm{O}
$$

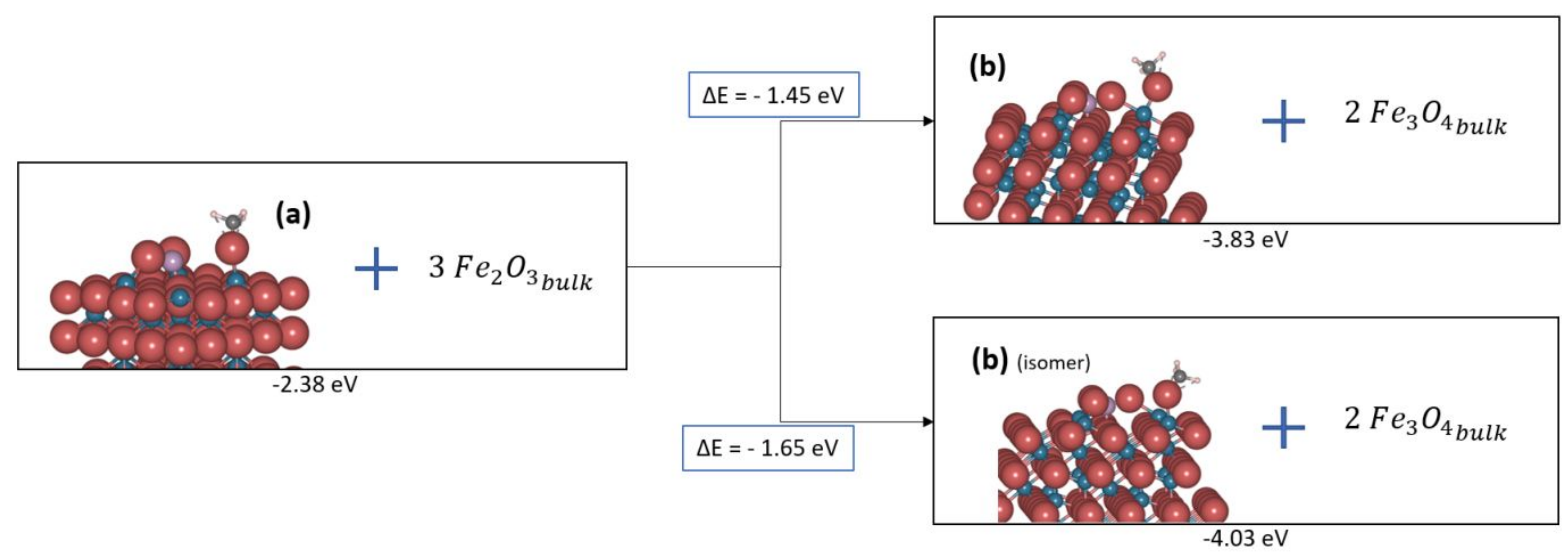

Fig. S6: Stabilization pathway of Intermediate a using O-adatom obtained from the reduction of hematite to produce Intermediate $b$. 


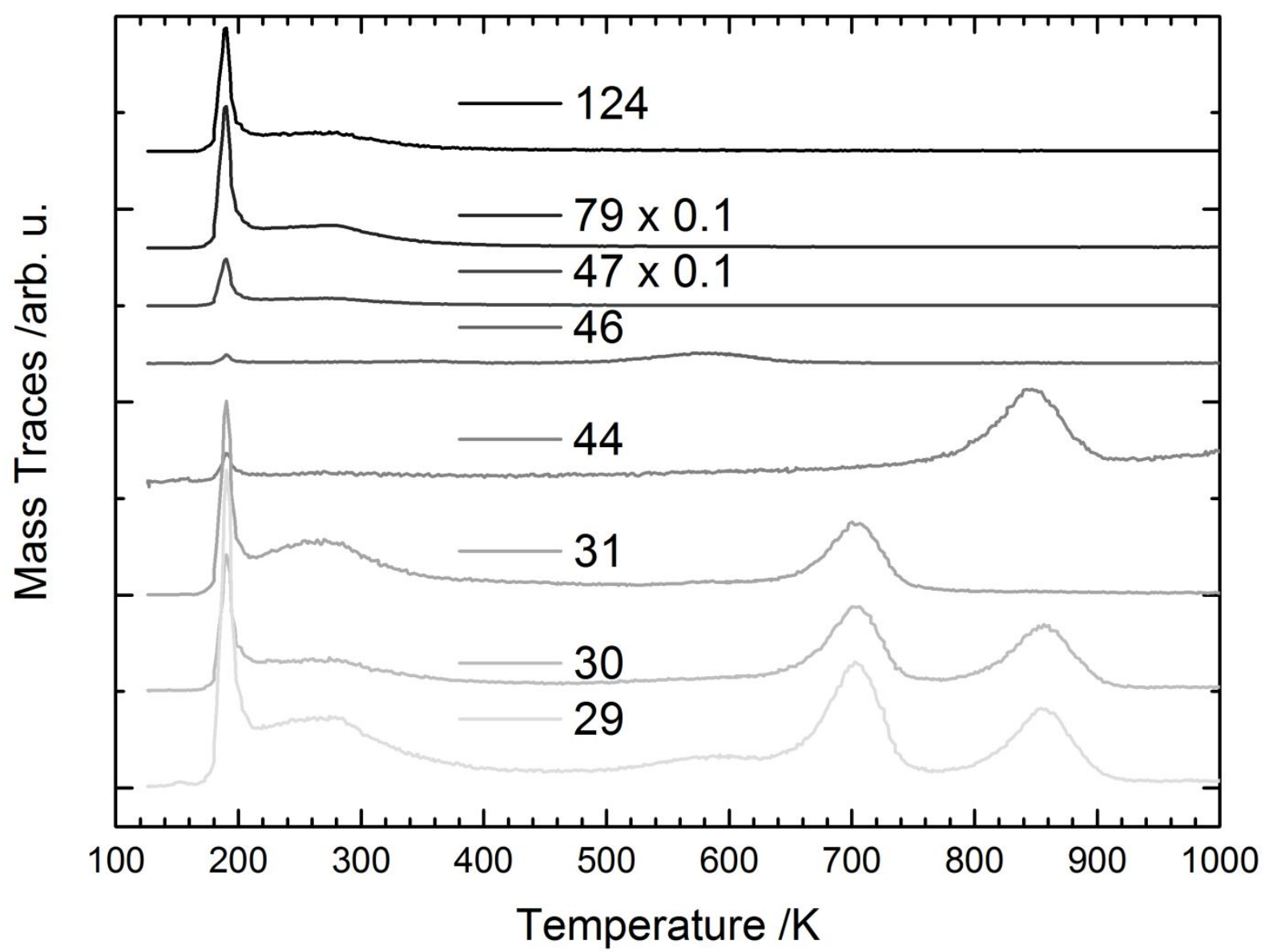

Fig. S7: Temperature-programmed desorption and reaction of dimethoxy methylphosphonate (DMMP) exposure $(60 \mathrm{~s})$ at $125 \mathrm{~K}$ on a freshly prepared $\mathrm{Fe}_{3} \mathrm{O}_{4}(111) / \mathrm{Fe}_{2} \mathrm{O}_{3}(0001)$ surface. Around $190 \mathrm{~K}$, a multilayer peak for DMMP is observed, indicated by masses 79 and the parent ion 124 in agreement with literature on a powdered hematite sample. ${ }^{8}$ The around $270 \mathrm{~K}$, present in all traces, indicates a reversible adsorption of DMMP below room temperature. The features above room temperatures are the same as in Fig. 1 and the absence peaks above $300 \mathrm{~K}$ of the mass traces 47, 79 and 124 indicates, that no $\mathrm{PO}_{x}$-species are desorbing. Based on a comparison in the observed intensities, the obtained high temperature reactivity is the same as on Fig. 1.

Table S4: Elemental Analysis of Desorbing Molecules from Table 1 and Figure 1. The assumption for the theoretical stoichiometry of the DMMP decomposition is a cleavage of all P-X single bonds from DMMP on the surface.

\begin{tabular}{c|c|c|c} 
Relative Abundance & $\mathrm{H}$ & $\mathrm{C}$ & $\mathrm{O}$ \\
\hline \hline Theoretical & 3.00 & 1.00 & 1.50 \\
\hline Experiment & 2.97 & 1.08 & 1.52
\end{tabular}


a) DMMP

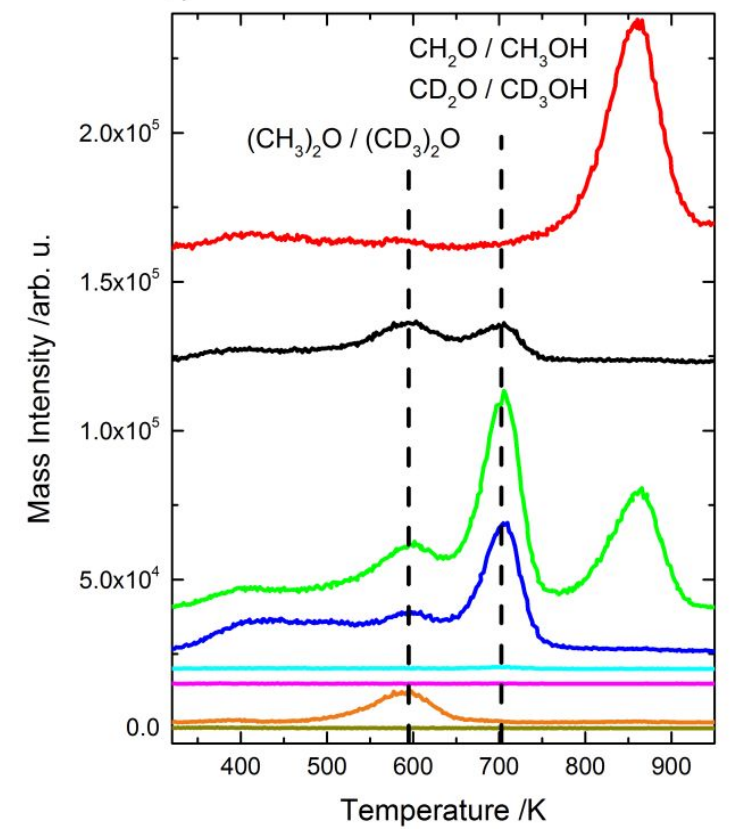

b) $\mathrm{DMMP}+\mathrm{CD}_{3} \mathrm{OH}$

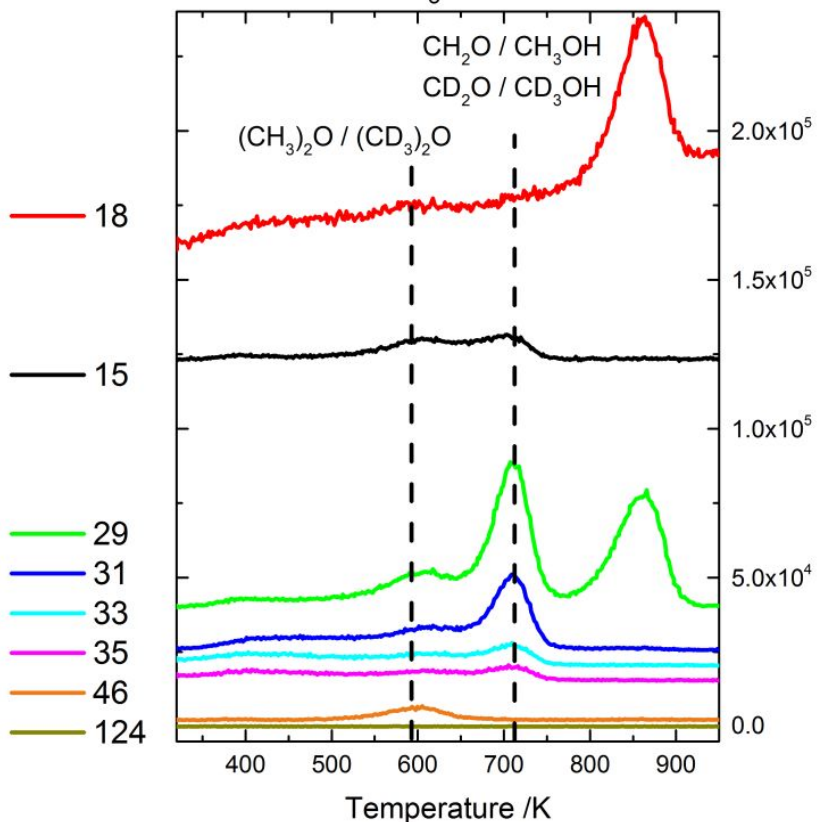

Fig. S8: Temperature programmed reaction of a DMMP saturated $\mathrm{Fe}_{3} \mathrm{O}_{4}(111) / \mathrm{Fe}_{2} \mathrm{O}_{3}(0001)$ surface (a) showing more raw mass traces confirming dimethyl ether, methanol, formaldehyde, water, $\mathrm{CO}$ and $\mathrm{CO}_{2}$ desorption. In part b), the surface is only exposed to DMMP for $10 \mathrm{~s}$ and afterwards for $60 \mathrm{~s}$ of $\mathrm{CD}_{3} \mathrm{OH}$ at $1.0 \times$ $10^{-9}$ Torr at room temperature. In the DME formation, especially the mass fragment 15 and 46 are prominent (a), while no mass 18 fragment is observed, indicating a DME formation pathway that does not involve surface methoxies. A detailed analysis of the mass traces 29-35 further shows, that isotopically labelled methanol is only observed in the surface methoxy decomposition feature and also absent in the combustion feature at $870 \mathrm{~K}$, corroborating the mechanism presented in Scheme 1. Note, that ehe traces are offset for clarity and no intact DMMP desorption is observed for a room temperature adsorption as indicated by the flat line of mass trace 124 , the parent mass of DMMP. 


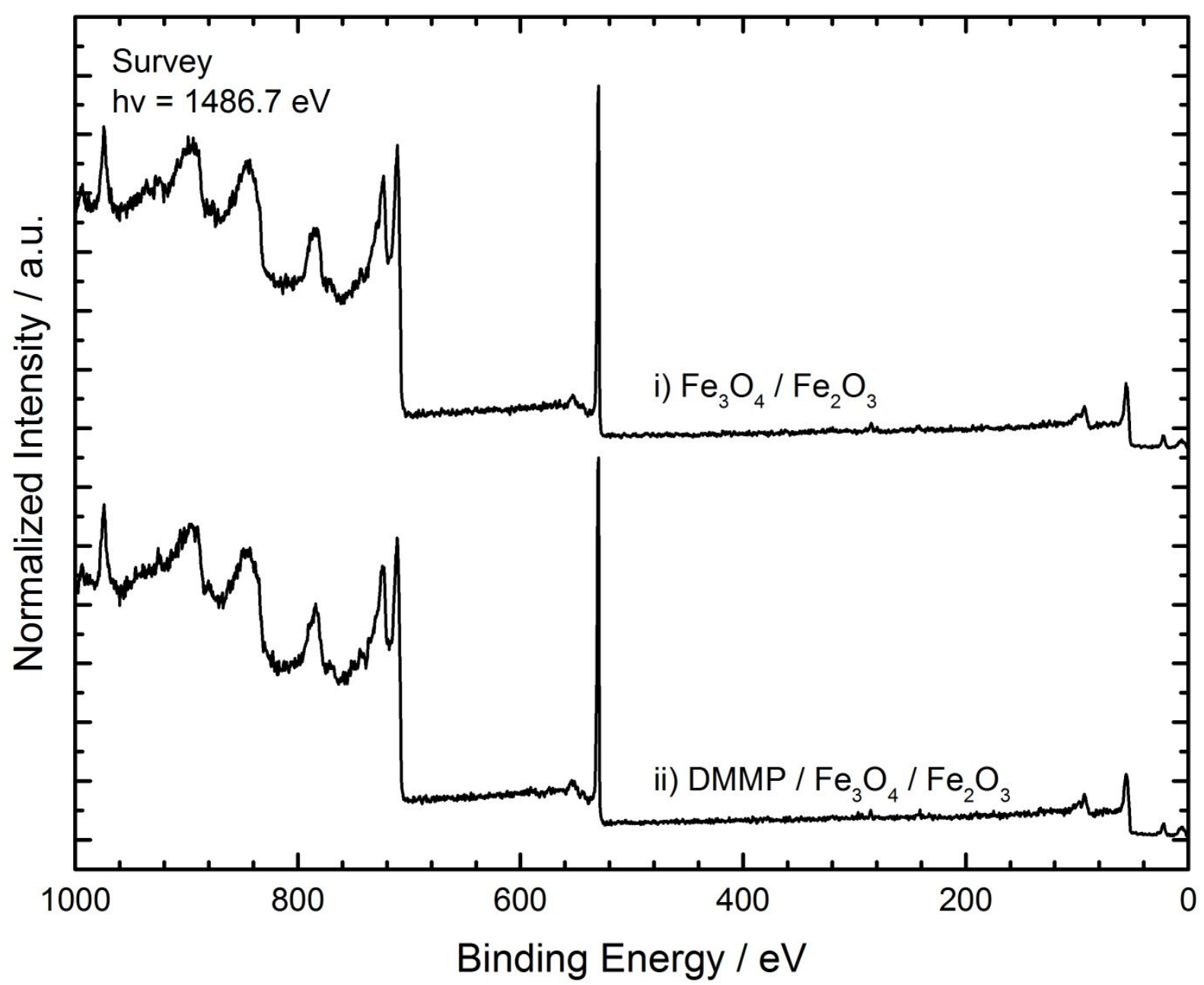

Fig. S9: Survey X-ray photoelectron spectra of the freshly prepared $\mathrm{Fe}_{3} \mathrm{O}_{4}(111) / \mathrm{Fe}_{2} \mathrm{O}_{3}(0001)$ surface (i) and the surface exposed to a room temperature saturation coverage of DMMP (ii). Detailed scans of the Fe2p region (700 eV - 750 $\mathrm{eV})$, the $\mathrm{O} 1 \mathrm{~s}$ region $(525 \mathrm{eV}-539 \mathrm{eV})$ and the $\mathrm{P} 2 \mathrm{p}$ region $(120 \mathrm{eV}-145 \mathrm{eV})$ were performed, to investigate the DMMP adsorption in more detail. 


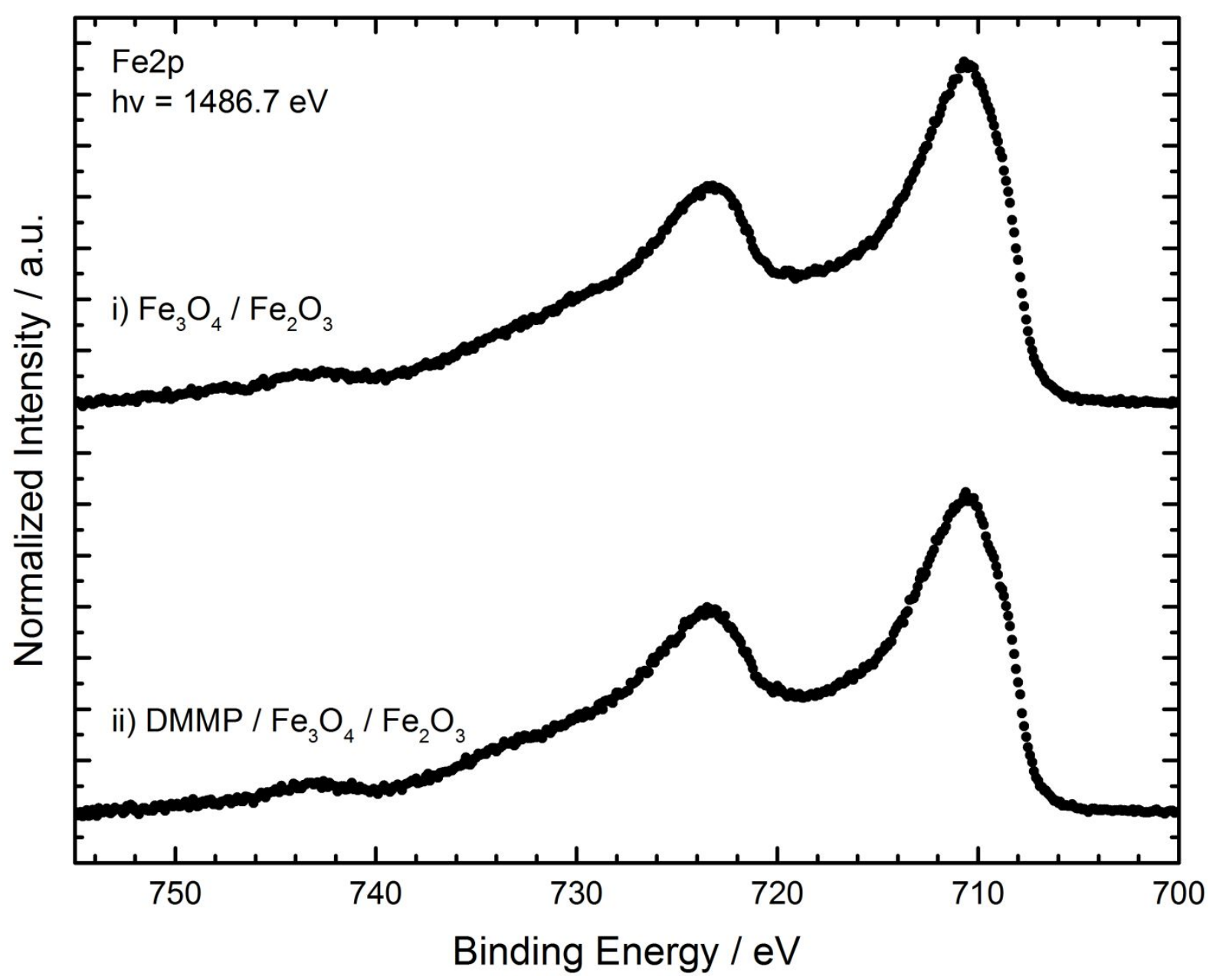

Fig. S10: XPS spectra acquired for the Fe2p region for the $\mathrm{Fe}_{3} \mathrm{O}_{4}(111) / \mathrm{Fe}_{2} \mathrm{O}_{3}(0001)$ surface (i) and the DMMP covered $\mathrm{Fe}_{3} \mathrm{O}_{4}(111) / \mathrm{Fe}_{2} \mathrm{O}_{3}(0001)$ surface (ii). Note that peak structure of the $\mathrm{Fe} 2 \mathrm{p}$ region with the two oxidation states and spin states is too complex to be fitted reliably. ${ }^{2}$ 


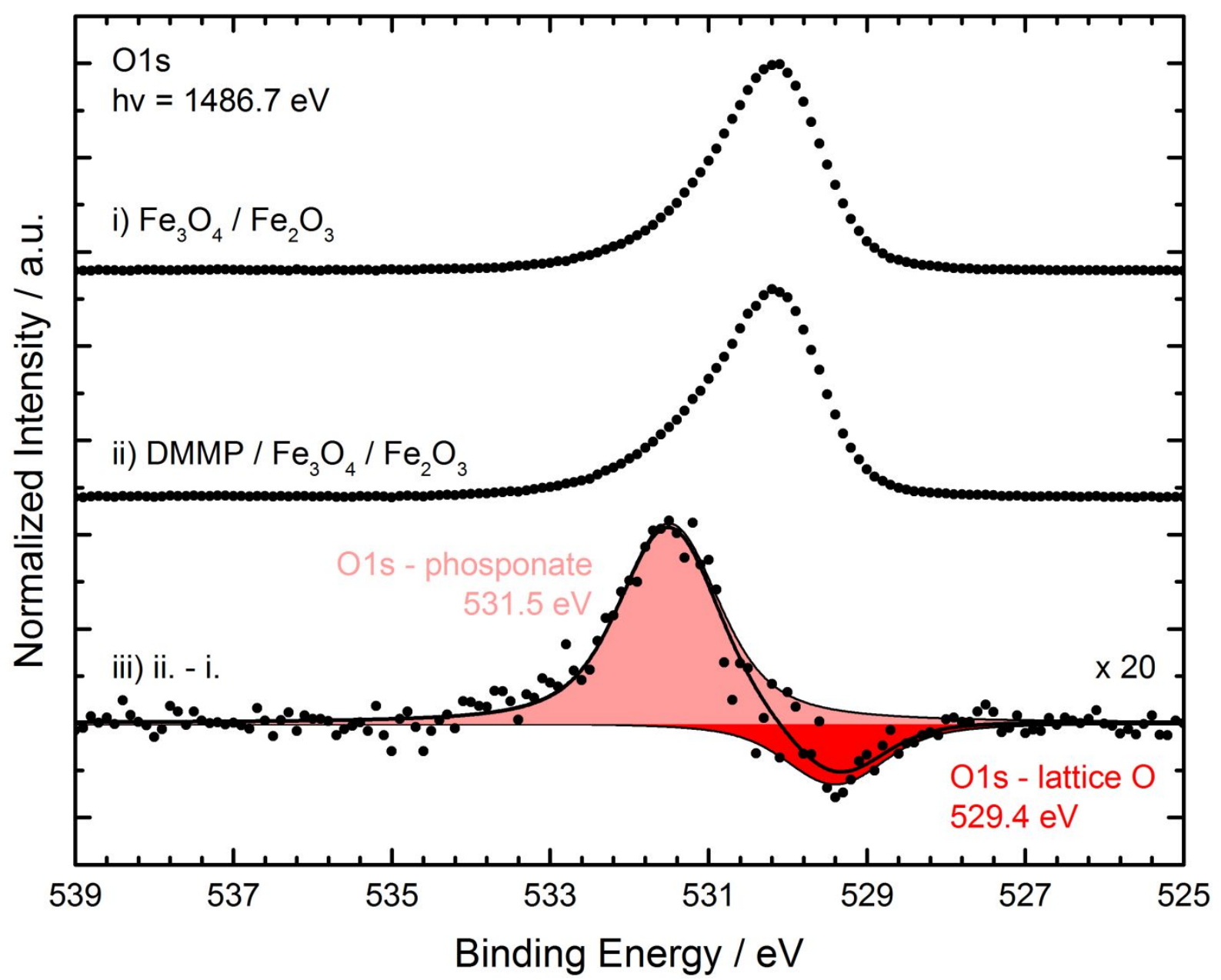

Fig. S11: XPS spectra acquired for the O1s region for the $\mathrm{Fe}_{3} \mathrm{O}_{4}(111) / \mathrm{Fe}_{2} \mathrm{O}_{3}(0001)$ surface (i), the DMMP covered $\mathrm{Fe}_{3} \mathrm{O}_{4}(111) / \mathrm{Fe}_{2} \mathrm{O}_{3}(0001)$ surface (ii) and the difference spectrum (iii) of the two above. In the difference spectrum (iii), a peak attributed to phosphorous bound oxygen is visible and located around $531.5 \mathrm{eV}$, that is assigned to a phosphorous bound oxygen species, while a attenuation in the lattice oxygen peak ${ }^{9-}$ 10 at $529.4 \mathrm{eV}$ is observed. 


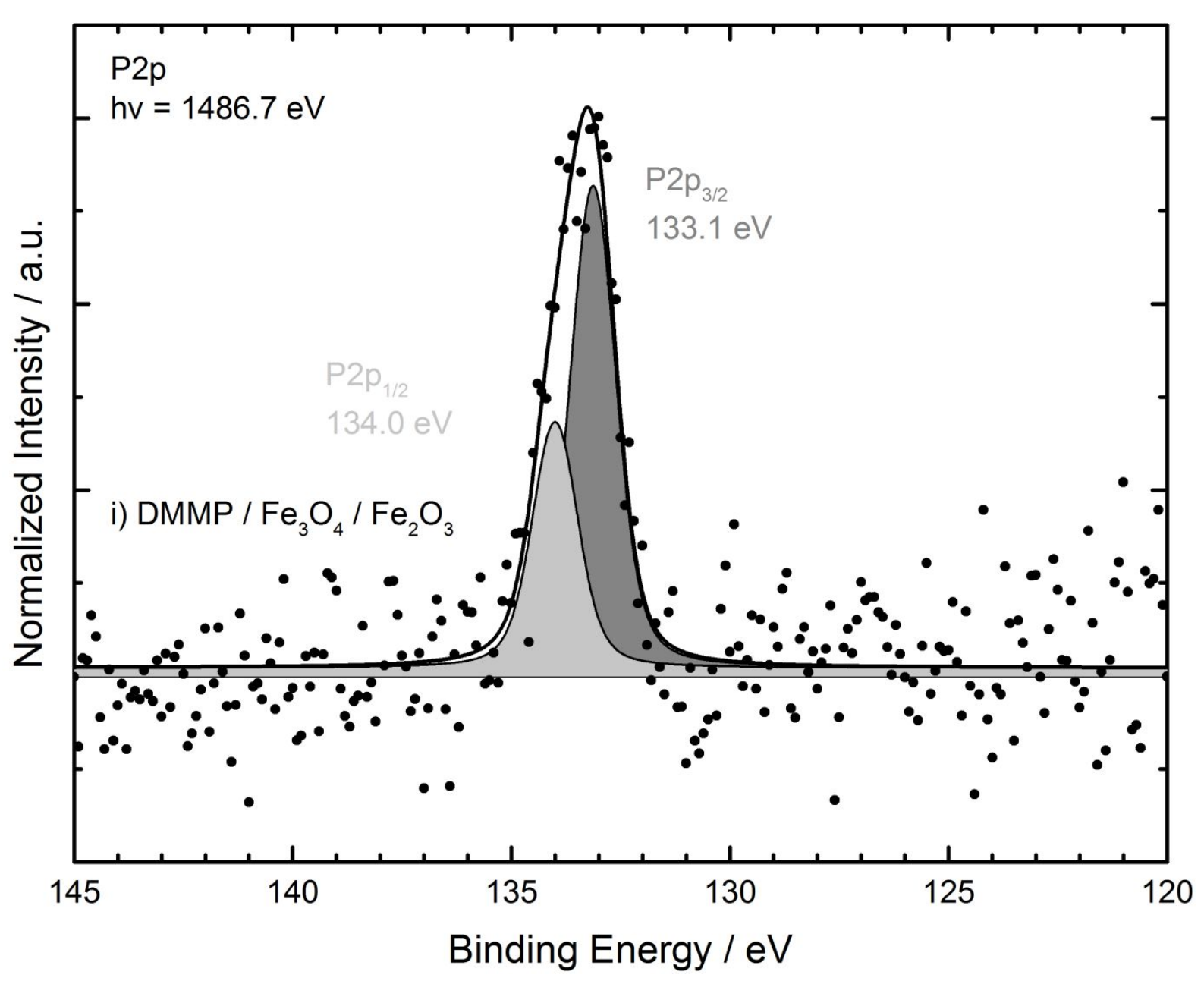

Fig. S12: XPS spectra acquired for the P2p region for the DMMP covered $\mathrm{Fe}_{3} \mathrm{O}_{4}(111) / \mathrm{Fe}_{2} \mathrm{O}_{3}(0001)$ surface. The energy splitting of $0.9 \mathrm{eV}$ the spin-orbit components in the $\mathrm{P} 2 \mathrm{p}$ spectrum is in quantitative agreement with a previous value $0.85 \mathrm{eV}$ measured for DMMP with ambient pressure XPS. ${ }^{11}$ The feature of the P2p region between binding energies of $131 \mathrm{eV}$ to $134 \mathrm{eV}$ is in very good agreement with work on $\mathrm{MoO}_{3}{ }^{12-13}$ and $\mathrm{Cu}_{2} \mathrm{O}^{14}$.

Tab. S5: Integrated Areas from Fig. S6 and S7 for a stoichiometry assignment of P and $O$ species on adsorbed on the surface. An estimate of the adsorbate stoichiometry is obtained by comparing the peal areas of the P-region with the 01s - phosphonate signal shows, that an O/P ratio of 2.91 is obtained for the adsorbate at $800 \mathrm{~K}$, which aligns very well with the proposed mechanism. Note, confidence interval for the O/P ratio is $\pm 10 \%$, since data processing can affect the exact ratio.

\begin{tabular}{|c|c|c|}
\hline & $P 2 p$ - total & O1s - phosphonate \\
\hline Total Area & 0.29 & 2.12 \\
\hline Atomic Sensitivity Factor ${ }^{15}$ & 0.25 & 0.63 \\
\hline Corrected Area & 1.16 & 3.37 \\
\hline
\end{tabular}




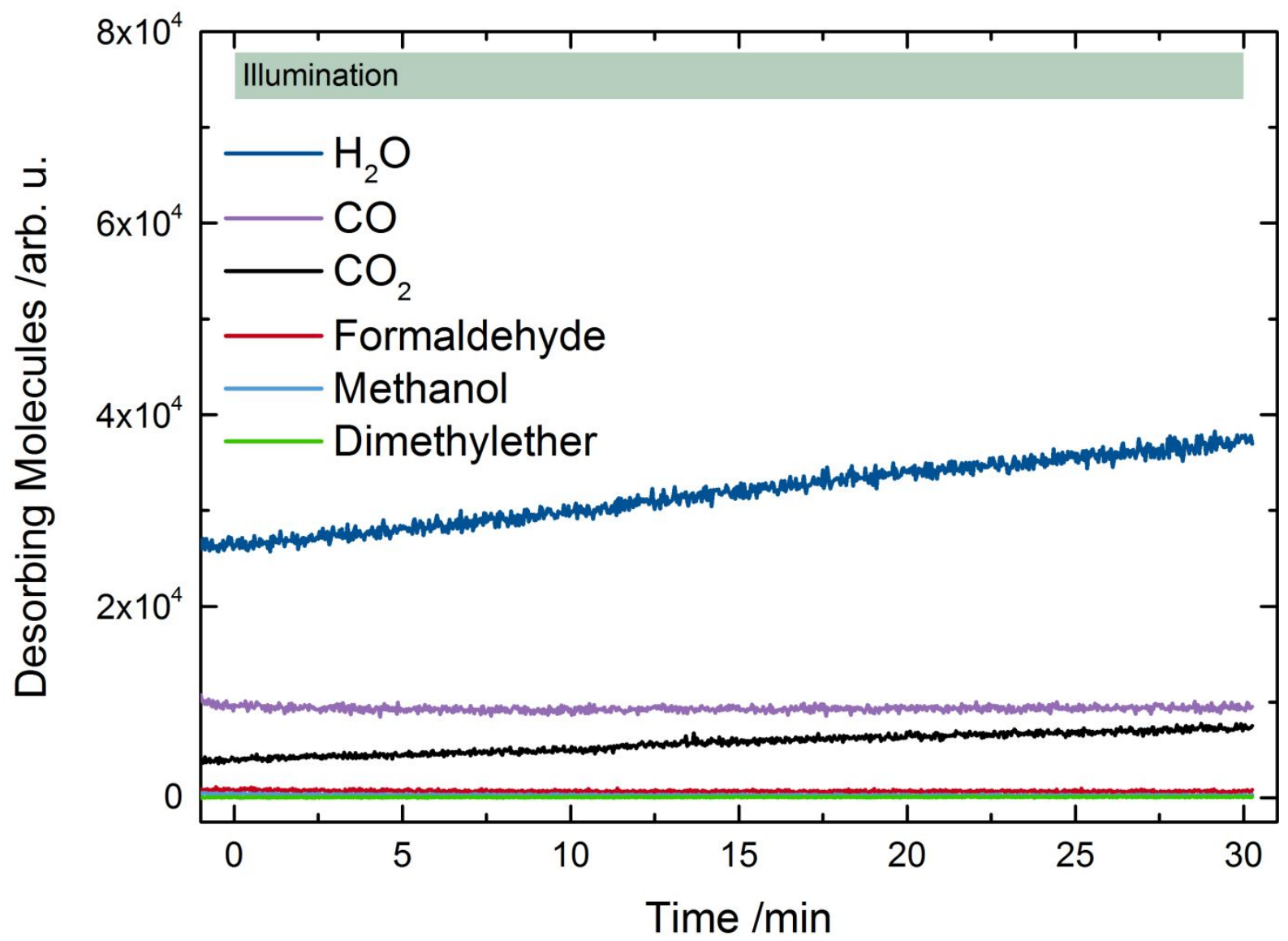

Fig. S13: Isothermal photoreaction at with UV light at $300 \mathrm{~K}$ with on a DMMP saturated $\mathrm{Fe}_{3} \mathrm{O}_{4}(111) / \mathrm{Fe}_{2} \mathrm{O}_{3}(0001)$ surface. A desorption in any of the monitored masses is not observed. The water constant rise in the background of water and carbon monoxide is attributed to desorption in the QMS filament during operation. The displayed masses are corrected for fragmentation and ionization and are the same as in Figure 1. 


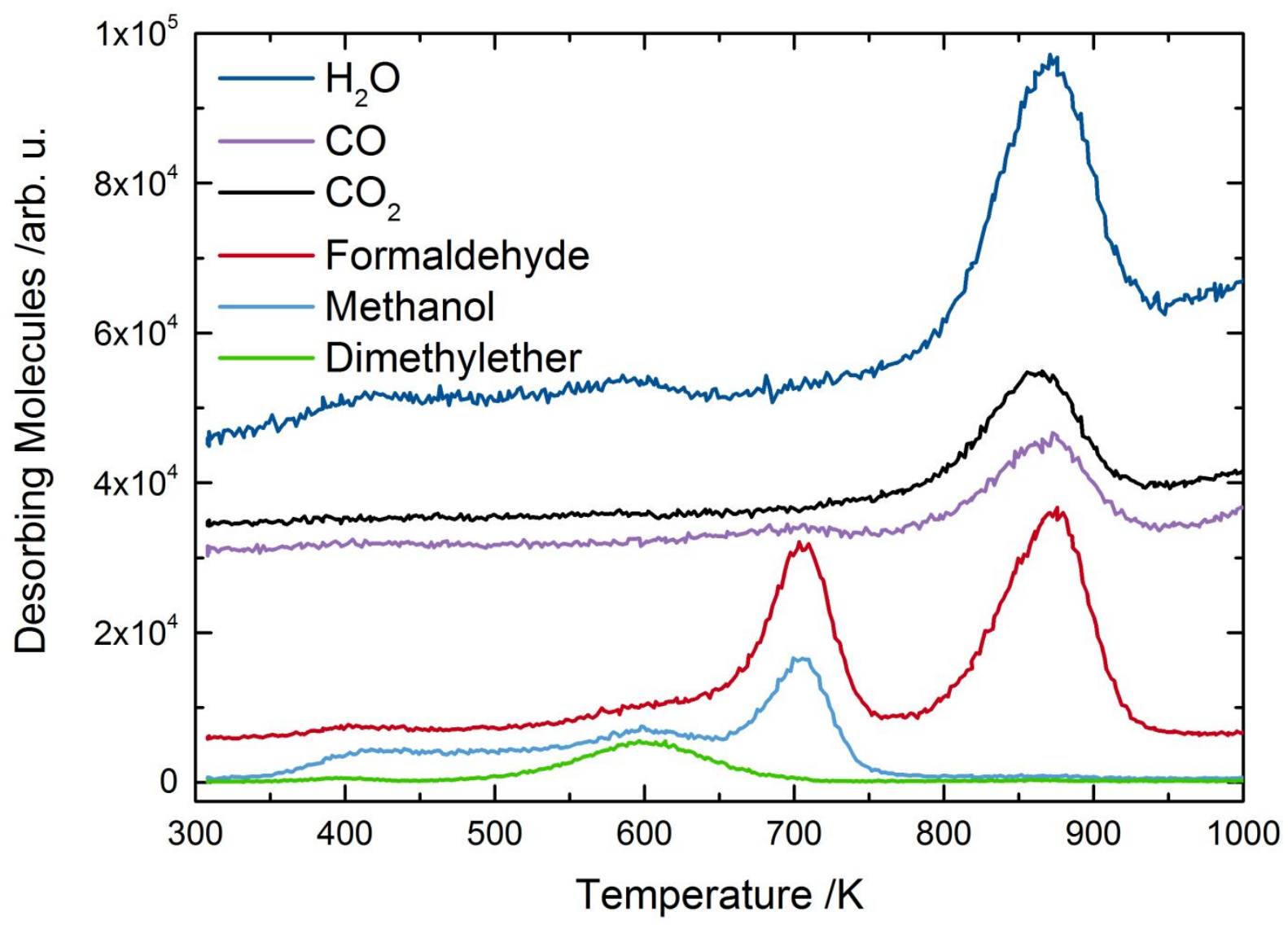

Fig. S14: Consecutive temperature-programmed reaction experiment of a room temperature saturation dose of DMMP after $30 \mathrm{~min}$ of UV illumination (Fig. S8). There are no additional products formed in comparison with a TPRS experiment without prior illumination (see Fig. 1 and Fig. S3a) and the product distribution is not changed. The displayed masses are corrected for fragmentation and ionization and are the same as in Figure 1. A control experiment using white light did also not result in any detectable photochemical reaction in a consecutive TPRS as well. 
Atomic Cooridnates:

\title{
Coordinates: Most stable molecular adsorption DMMP index (2)
}

\author{
$\mathrm{CFe} \mathrm{HOP}$ \\ 1.00000000000000 \\ $\begin{array}{llll}11.9218206406000000 & 0.0000000000000000 & 0.0000000000000000\end{array}$ \\ $\begin{array}{llll}5.9609103203000000 & 10.3245995341000008 & 0.0000000000000000\end{array}$ \\ $\begin{array}{lll}0.0000000000000000 & 0.0000000000000000 & 30.0000000000000000\end{array}$ \\ $\mathrm{C} \mathrm{Fe} \mathrm{H} \quad \mathrm{O} \quad \mathrm{P}$ \\ $\begin{array}{lllll}3 & 48 & 9 & 68 & 1\end{array}$
}

Selective dynamics

Direct

\begin{tabular}{|c|c|c|c|c|}
\hline & & & & \\
\hline & & & & \\
\hline 7761126305930283 & 3676510031805764 & 0.5200855995104061 & & \\
\hline 3367790249999985 & 3367790169999978 & 0.0327748283333378 & & \\
\hline & 8367790040000003 & 0.0327748283333378 & & \\
\hline & & & & \\
\hline & & & & \\
\hline & & 3359 & & \\
\hline 1123630000012 & 123409999994 & 0.0530542513333359 & & \\
\hline 6701123019999997 & 123429999996 & 0.053054251333 & & \\
\hline 123420000030 & 99996 & 0.05305425133 & & \\
\hline & & 0.112037 & & \\
\hline 738180630173 & 739739 & 0.11203779800 & & \\
\hline & & 782 & & \\
\hline & & 782 & & \\
\hline 37040630149 & & 782 & & \\
\hline 37570630158 & 30144 & 0.1120377980 & $\mathrm{~F}$ & \\
\hline & 0.4 & 0.112 & & \\
\hline & & & & \\
\hline & & 0.11203 & & \\
\hline 59 & 0.6 & 0.11203 & & \\
\hline & & 782 & & \\
\hline 630183 & 169 & 0.11 & & \\
\hline & & 732 & $\mathrm{~T}$ & \\
\hline & & 0.17 & 7 & \\
\hline & & 0.17 & & \\
\hline 62325640700 & 86094 & 0.17 & & \\
\hline & 0.0 & 0.1 & & \\
\hline & & 228 & & \\
\hline & & 982 & & \\
\hline & & & & \\
\hline 16674406535 & 8862134 & 0.214133 & $\mathrm{~T}$ & $\mathrm{~T}$ \\
\hline 731722319 & 18763832 & 0.21376 & & \\
\hline 90658031106 & 494832029125 & 0.21461 & & \\
\hline 92106527484 & 456748 & 0.21485 & & \\
\hline & & 0.278255 & & \\
\hline & & 3186 & & \\
\hline & 5934933523625 & 0.2766861356432613 & & \\
\hline 5865343957419866 & 3361718471495827 & 0.2767076383886882 & & \\
\hline 03348511424789870 & .5856318963912388 & 0.2771132012036263 & & \\
\hline
\end{tabular}


$\begin{array}{lllllll}0.5884949351035983 & 0.5874019014986035 & 0.2762642580133558 & \text { T } & \text { T } & \text { T }\end{array}$ $\begin{array}{lllllll}0.0871261115481547 & 0.0892171744250548 & 0.2771165840967322 & \text { T } & \text { T }\end{array}$ $\begin{array}{lllllll}0.0884907847803002 & 0.8339995433764721 & 0.2777603194473880 & \text { T } & \text { T }\end{array}$ $\begin{array}{llllll}0.5884789860404671 & 0.8340468650478401 & 0.2776431071718702 & \text { T } & \text { T }\end{array}$ $\begin{array}{lllllll}0.8360486571240551 & 0.5872915935464447 & 0.2759971336356634 & \text { T } & \text { T }\end{array}$ $\begin{array}{lllllll}0.8341094483527380 & 0.0906115598761290 & 0.2775263288040226 & \text { T } & \text { T }\end{array}$ $\begin{array}{lllllll}0.3342134386005571 & 0.0905293661388897 & 0.2778751020599136 & \text { T } & \text { T }\end{array}$ $\begin{array}{llllllll}0.3365902401077270 & 0.8377094681413770 & 0.3188500512988940 & \text { T } & \text { T }\end{array}$ $\begin{array}{lllllll}0.8360664385515053 & 0.8387033430728864 & 0.3186125961843332 & \text { T } & \text { T }\end{array}$ $\begin{array}{lllllll}0.8376414069872299 & 0.3394948185730537 & 0.3298240089293334 & \text { T } & \text { T }\end{array}$ $\begin{array}{lllllll}0.3359080633214384 & 0.3361426209254236 & 0.3338438883494984 & \text { T } & \text { T }\end{array}$ $\begin{array}{lllllllll}0.5330230462071217 & 0.4517046975422080 & 0.3783911536362454 & \text { T } & \text { T }\end{array}$ $\begin{array}{llllllll}0.5598577612919173 & 0.3006089693576814 & 0.4016492606128710 & \text { T } & \text { T }\end{array}$ $\begin{array}{llllllll}0.7790948927094897 & 0.1358279908058794 & 0.3993118395628820 & \text { T } & \text { T }\end{array}$ $\begin{array}{lllllll}0.4407565269645529 & 0.4491938108997930 & 0.4256364056102906 & \text { T } & \text { T }\end{array}$ $\begin{array}{lllllll}0.9272579820315474 & 0.0916641464936908 & 0.4273568593747752 & \text { T } & \text { T }\end{array}$ $\begin{array}{lllllll}0.7866009806011525 & 0.1208087751251998 & 0.4589442279734678 & \text { T } & \text { T }\end{array}$ $\begin{array}{lllllll}0.6723429042732887 & 0.4379855134895571 & 0.5174372821804651 & \text { T } & \text { T }\end{array}$ $\begin{array}{llllllll}0.7909667249322856 & 0.2769020479597909 & 0.5349183973825017 & \text { T } & \text { T }\end{array}$ $\begin{array}{lllllll}0.8244320113853925 & 0.4073358336589072 & 0.5404028543779467 & \text { T } & \text { T } & \text { T }\end{array}$ $\begin{array}{llllll}0.7568914029999994 & 0.4965543409999995 & 0.0741721933333395 & F & F & F\end{array}$ $0.75689140600000120 .75689134099999930 .0741721933333395 \quad F \quad F \quad F$ $\begin{array}{llllll}0.4965543189999977 & 0.7568913409999993 & 0.0741721933333395 & F & F & F\end{array}$ $\begin{array}{llllll}0.4965542930000026 & 0.2568913539999969 & 0.0741722013333330 & F & F & F\end{array}$ $\begin{array}{llllll}0.2568913460000033 & 0.4965542950000028 & 0.0741722013333330 & F & F & F\end{array}$ $\begin{array}{lllllll}0.2568914200000023 & 0.2568913539999969 & 0.0741722013333330 & F & F & F\end{array}$ $\begin{array}{llllll}0.9965543819999994 & 0.7568912950000026 & 0.0741722013333330 & F & F & F\end{array}$ $0.25689138900000330 .99655432800000200 .0741722013333330 \quad F \quad F \quad F$ $0.7568912999999995 \quad 0.25689135399999690 .0741722013333330 \quad F \quad F \quad F$ $\begin{array}{llllll}0.9965543329999988 & 0.2568913539999969 & 0.0741722013333330 & F & F & F\end{array}$ $\begin{array}{llllll}0.7568913490000000 & 0.9965543280000020 & 0.0741722013333330 & F & F & F\end{array}$ $\begin{array}{lllllll}0.2568913890000033 & 0.7568912950000026 & 0.0741722013333330 & F & F & F\end{array}$ $\begin{array}{lllllll}0.0034456799999987 & 0.0034456819999988 & 0.0758492213333355 & F & F & F\end{array}$ $\begin{array}{lllllll}0.5034457029999970 & 0.0034456619999972 & 0.0758492373333368 & F & F & F\end{array}$ $0.00344567399999820 .50344567900000210 .0758492373333368 \quad F \quad F \quad F$ $\begin{array}{lllllll}0.5034457940000010 & 0.5034456790000021 & 0.0758492373333368 & F & F & F\end{array}$ $\begin{array}{lllllll}0.3367790249999985 & 0.3367790169999978 & 0.1500589058244657 & F & F & F\end{array}$ $\begin{array}{llllll}0.3367789719999976 & 0.8367790040000003 & 0.1500589218244670 & F & F & F\end{array}$ $\begin{array}{llllll}0.8367790920000004 & 0.8367790040000003 & 0.1500589218244670 & F & F & F\end{array}$ $\begin{array}{llllll}0.8367789970000032 & 0.3367789939999994 & 0.1500589218244670 & F & F & F\end{array}$ $\begin{array}{lllllll}0.8454804489765664 & 0.5824282795117171 & 0.1517299961083083 & F & F & F\end{array}$ $\begin{array}{lllllll}0.5824282575117152 & 0.5824282795117171 & 0.1517299961083083 & F & F & F\end{array}$ $\begin{array}{llllll}0.5824282385117172 & 0.8454805109765644 & 0.1517299961083083 & F & F & F\end{array}$ $0.0824282805117207 \quad 0.58242823351172040 .1517300121083096 \quad F \quad F \quad F$ $\begin{array}{lllllll}0.3454805459765637 & 0.0824282865117141 & 0.1517300121083096 & F & F & F\end{array}$ $\begin{array}{llllll}0.5824282745117202 & 0.0824282865117141 & 0.1517300121083096 & F & F & F\end{array}$ $\begin{array}{lllllll}0.0824283145117164 & 0.3454804769765616 & 0.1517300121083096 & F & F & F\end{array}$ $\begin{array}{llllll}0.3454805119765680 & 0.5824282335117204 & 0.1517300121083096 & F & F & F\end{array}$ $\begin{array}{lllllll}0.8454804259765680 & 0.0824282865117141 & 0.1517300121083096 & F & F & F\end{array}$ $\begin{array}{lllllll}0.0824283245117172 & 0.8454804179765674 & 0.1517300121083096 & F & F & F\end{array}$ $\begin{array}{lllllll}0.0824282945117147 & 0.0824282865117141 & 0.1517300121083096 & F & F & F\end{array}$ $\begin{array}{lllllll}0.5824282345117169 & 0.3454804769765616 & 0.1517300121083096 & F & F & F\end{array}$ $\begin{array}{lllllll}0.9253146235943126 & 0.4249673070369875 & 0.2310429827941413 & \text { T } & \text { T }\end{array}$ 


\begin{tabular}{|c|c|c|c|c|}
\hline $\cos ^{\circ}$ & 0.6608959133348900 & & $\mathrm{~T}$ & \\
\hline 6610654819717358 & 0.9255923398757472 & 0.2321125213744721 & $\mathrm{~T}$ & \\
\hline .4245603532811127 & 0.9251194776354922 & 0.2320636312392495 & $\mathrm{~T}$ & \\
\hline 9251102947906514 & 0.6601962135345357 & 0.2316905311208981 & $\mathrm{~T}$ & \\
\hline 6612031060985031 & 0.4242218530701287 & 0.2313876604592200 & $\mathrm{~T}$ & \\
\hline 240799713262005 & 0.9259161435605004 & 0.2323007984607847 & $\mathrm{~T}$ & \\
\hline 1620818002790337 & 0.4242724113141961 & 0.2312293114948030 & $\mathrm{~T}$ & \\
\hline 0.1601813133441349 & 0.9256475014662943 & 0.2321534129572370 & T & \\
\hline 0.9239749431469998 & 0.1618105645229752 & 0.2316508765653149 & $\mathrm{~T}$ & \\
\hline 0.4259375378784338 & 0.4248292087033886 & 0.2314733791416149 & $\mathrm{~T}$ & \\
\hline 0.4253886182920182 & 0.1611040677686367 & 0.2315305989169497 & $\mathrm{~T}$ & \\
\hline .6700322112844256 & 0.6702241233282568 & 0.2349319000581671 & $\mathrm{~T}$ & \\
\hline 6700978347958049 & 0.1715823191956724 & 0.2358292036076648 & $\mathrm{~T}$ & \\
\hline 0.1697113734097961 & 0.1709377713682081 & 0.2353819348227766 & $\mathrm{~T}$ & \\
\hline 0.1705440459035043 & 0.6697112056427198 & 0.2353253739069677 & $\mathrm{~T}$ & $\mathrm{~T}$ \\
\hline 0.0026839420844248 & 0.0050612233473548 & 0.3058113422467577 & $\mathrm{~T}$ & \\
\hline 0.0034688447714394 & 0.5030269903117731 & 0.3050685548713654 & $\mathrm{~T}$ & \\
\hline 0.5036973388232805 & 0.0038836199264598 & 0.3055442418973415 & $\mathrm{~T}$ & \\
\hline 0.5046889113592510 & 0.5035151224891525 & 0.3055648181579823 & $\mathrm{~T}$ & \\
\hline 2503824930879973 & 0.7471920898847479 & 0.3090287983317354 & $\mathrm{~T}$ & \\
\hline .7478704104939217 & 0.0169880912052847 & 0.3101571338532081 & $\mathrm{~T}$ & $\mathrm{~T}$ \\
\hline 0.0024559739803498 & 0.2554382270535497 & 0.3068585372099525 & $\mathrm{~T}$ & $\mathrm{~T}$ \\
\hline 0.7508255042561811 & 0.7473259265550021 & 0.3084714433856754 & $\mathrm{~T}$ & $\mathrm{~T}$ \\
\hline 0.2559609901765934 & 0.4996193098781821 & 0.3067949114322259 & $\mathrm{~T}$ & \\
\hline 0.2524794459566332 & 0.2559885368827433 & 0.3066499631868581 & $\mathrm{~T}$ & \\
\hline 0.7547721520706721 & 0.5021235630089846 & 0.3057140589230338 & $\mathrm{~T}$ & \\
\hline 0.5134895484634917 & 0.7469603625918586 & 0.3088603248565427 & $\mathrm{~T}$ & $\mathrm{~T}$ \\
\hline 0.5019957698060524 & 0.2572289500571504 & 0.3073943905100255 & $\mathrm{~T}$ & $\mathrm{~T}$ \\
\hline 0.0127069444887299 & 0.7478565228533495 & 0.3089615578677505 & $\mathrm{~T}$ & $\mathrm{~T}$ \\
\hline 0.2471263244016126 & 0.0151496920234574 & 0.3092672598873531 & $\mathrm{~T}$ & $\mathrm{~T}$ \\
\hline 0.7526635765268781 & 0.2563038474981175 & 0.3084067786967548 & $\mathrm{~T}$ & $\mathrm{~T}$ \\
\hline 0.3510791170809578 & 0.3097713752739680 & 0.3884719108984484 & $\mathrm{~T}$ & $\mathrm{~T}$ \\
\hline 0.8313461794855967 & 0.3535127376498235 & 0.3942087026518222 & $\mathrm{~T}$ & $\mathrm{~T}$ \\
\hline 0.6275868191727969 & 0.4027261784098023 & 0.4407924278050367 & $\mathrm{~T}$ & $\mathrm{~T}$ \\
\hline 0.8366438621004324 & 0.3428677712129562 & 0.4766877504927346 & $\mathrm{~T}$ & \\
\hline 0.7776978970112525 & 0.3167908485373153 & 0.4334703427603395 & & $\mathrm{~T}$ \\
\hline
\end{tabular}

\section{Coordinates: Most stable dissociative adsorption MMP and methoxy (5)}

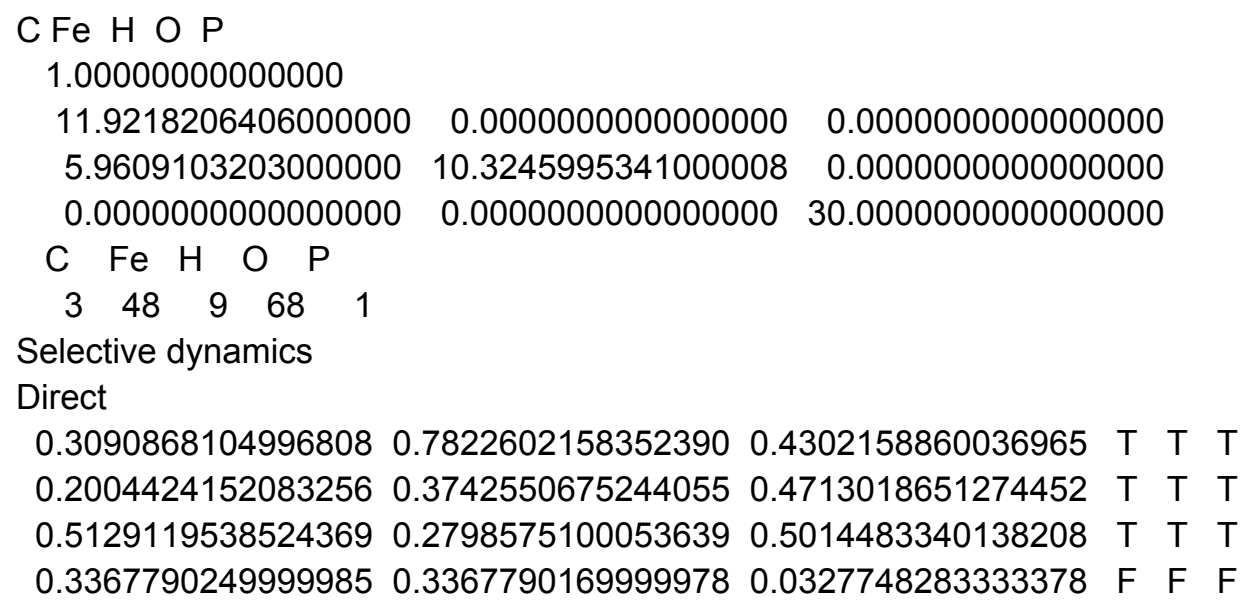


$\begin{array}{llllll}0.3367789719999976 & 0.8367790040000003 & 0.0327748283333378 & F & F & F\end{array}$ $\begin{array}{lllllll}0.8367789850000023 & 0.3367790169999978 & 0.0327748283333378 & F & F & F\end{array}$ $\begin{array}{llllll}0.8367790920000004 & 0.8367790040000003 & 0.0327748283333378 & F & F & F\end{array}$ $0.67011232299999790 .67011234099999940 .0530542513333359 \quad F \quad F \quad F$ $0.17011236300000120 .67011234099999940 .0530542513333359 \quad F \quad F \quad F$ $\begin{array}{lllllll}0.6701123019999997 & 0.1701123429999996 & 0.0530542513333359 & F & F & F\end{array}$ $\begin{array}{lllll}0.1701123420000030 & 0.1701123429999996 & 0.0530542513333359 & F & F\end{array}$ $\begin{array}{llllll}0.1705895288739754 & 0.4198737410630144 & 0.1120377980019782 & F & F & F\end{array}$ $\begin{array}{lllllll}0.9198738180630173 & 0.1705895108739739 & 0.1120377980019782 & F & F & F\end{array}$ $\begin{array}{llllll}0.4198737380630178 & 0.1705895108739739 & 0.1120377980019782 & F & F & F\end{array}$ $\begin{array}{lllllll}0.1705895158739779 & 0.9198737280630169 & 0.1120377980019782 & F & F & F\end{array}$ $0.91987370406301490 .91987372806301690 .1120377980019782 \quad F \quad F \quad F$ $\begin{array}{llllll}0.4198737570630158 & 0.4198737410630144 & 0.1120377980019782 & F & F & F\end{array}$ $\begin{array}{lllllll}0.9198738370630153 & 0.4198737410630144 & 0.1120377980019782 & F & F & F\end{array}$ $\begin{array}{lllllll}0.6705894888739721 & 0.4198737410630144 & 0.1120377980019782 & F & F & F\end{array}$ $0.67058951587397790 .91987372806301690 .1120377980019782 \quad F \quad F \quad F$ $0.91987375906301590 .67058950887397370 .1120377980019782 \quad F \quad F \quad F$ $0.41987371906301970 .67058950887397370 .1120377980019782 \quad F \quad F \quad F$ $\begin{array}{llllll}0.4198737440630183 & 0.9198737280630169 & 0.1120377980019782 & F & F & F\end{array}$ $\begin{array}{lllllll}0.1710736802913156 & 0.6700087521646301 & 0.1737052673968054 & \text { T } & \text { T }\end{array}$ $\begin{array}{lllllll}0.6692662705358525 & 0.6709814671172131 & 0.1736072788767707 & \text { T } & \text { T }\end{array}$ $\begin{array}{lllllll}0.1701369986936394 & 0.1693124537710641 & 0.1734809986386212 & \text { T } & \text { T }\end{array}$ $\begin{array}{lllllll}0.6700117879651050 & 0.1702124805951475 & 0.1733116115930758 & \text { T } & \text { T }\end{array}$ $\begin{array}{lllllll}0.5062130352965776 & 0.0022355591355871 & 0.1946755837669561 & \text { T } & \text { T }\end{array}$ $\begin{array}{lllllll}0.5027016808816336 & 0.5021257106549745 & 0.1947396379052777 & \text { T } & \text { T }\end{array}$ $\begin{array}{lllllll}0.0019533520751839 & 0.0056757479973908 & 0.1948944230860593 & \text { T } & \text { T }\end{array}$ $\begin{array}{lllllll}0.0029230712052405 & 0.5038861892816300 & 0.1954269661376853 & \text { T } & \text { T }\end{array}$ $\begin{array}{lllllll}0.3370291176512832 & 0.3360577697138318 & 0.2144562384079770 & \text { T } & \text { T } & \text { T }\end{array}$ $\begin{array}{lllllll}0.8358052526787815 & 0.3373652800904522 & 0.2153188011719378 & \text { T } & \text { T }\end{array}$ $\begin{array}{lllllll}0.3369845253655416 & 0.8366449422866250 & 0.2150072061667780 & \text { T } & \text { T }\end{array}$ $\begin{array}{lllllll}0.8370718480490835 & 0.8370952916819832 & 0.2153189112213276 & \text { T } & \text { T }\end{array}$ $\begin{array}{llllll}0.5856664492093404 & 0.0902422594332184 & 0.2777229336346352 & \text { T } & \text { T } & \text { T }\end{array}$ $\begin{array}{lllllll}0.0907011756796912 & 0.5861175609436942 & 0.2781028980556204 & \text { T } & \text { T }\end{array}$ $\begin{array}{lllllll}0.0899549727354480 & 0.3338890812989503 & 0.2776689541900539 & \text { T } & \text { T }\end{array}$ $\begin{array}{llllllll}0.5859326812929019 & 0.3348328258286107 & 0.2777222372755634 & \text { T } & \text { T }\end{array}$ $\begin{array}{lllllll}0.3345543205338044 & 0.5882813686041696 & 0.2780649053969329 & \text { T } & \text { T }\end{array}$ $\begin{array}{llllll}0.5851114293723398 & 0.5905228423262133 & 0.2777242275680544 & \text { T } & \text { T }\end{array}$ $\begin{array}{llllll}0.0908298904508090 & 0.0858751728177825 & 0.2780517242319750 & \text { T } & \text { T }\end{array}$ $\begin{array}{llllll}0.0907429886026094 & 0.8341799900933198 & 0.2778928263196042 & \text { T } & \text { T }\end{array}$ $\begin{array}{lllllll}0.5853045599603064 & 0.8346758798640905 & 0.2780670278530946 & \text { T } & \text { T }\end{array}$ $\begin{array}{lllllll}0.8333926287038194 & 0.5886988083859456 & 0.2781475071008700 & \text { T } & \text { T }\end{array}$ $\begin{array}{lllllll}0.8336882889037520 & 0.0882710146697935 & 0.2779510929509184 & \text { T } & \text { T }\end{array}$ $\begin{array}{lllllll}0.3349794961987757 & 0.0870699722993169 & 0.2772047738599671 & \text { T } & \text { T }\end{array}$ $\begin{array}{llllll}0.8377868667216284 & 0.3364102971639018 & 0.3199452487509428 & \text { T } & \text { T }\end{array}$ $\begin{array}{lllllll}0.8380039826245953 & 0.8365362279455555 & 0.3200809847264345 & \text { T } & \text { T }\end{array}$ $\begin{array}{lllllll}0.3362346186358472 & 0.3361191443546862 & 0.3335289220677277 & \text { T } & \text { T }\end{array}$ $\begin{array}{lllllll}0.3356807288530108 & 0.8375292721484786 & 0.3350212887873231 & \text { T } & \text { T }\end{array}$ $\begin{array}{llllll}0.3192294427287197 & 0.6883055622220543 & 0.4207497637366042 & \text { T } & \text { T }\end{array}$ $\begin{array}{lllllll}0.2100524477307926 & 0.8451422007367091 & 0.4430878571806567 & \text { T } & \text { T }\end{array}$ $\begin{array}{llllll}0.3784609562366069 & 0.7654410853147197 & 0.4572892055336276 & \text { T } & \text { T }\end{array}$ $\begin{array}{lllllll}0.1072069304755000 & 0.4486669193600676 & 0.4575825818705305 & \text { T } & \text { T }\end{array}$ $\begin{array}{lllllll}0.2087981304559835 & 0.2786643765376777 & 0.4680478120757219 & \text { T } & \text { T }\end{array}$ $\begin{array}{llllllll}0.5140526870463036 & 0.3713816601239902 & 0.4997223678287088 & \text { T } & \text { T }\end{array}$ 
$\begin{array}{llllll}0.2047988491237853 & 0.3958437994492995 & 0.5066464199402729 & \text { T } & \text { T }\end{array}$ $\begin{array}{lllllll}0.6118451150085835 & 0.1980876156180969 & 0.5049392293408018 & \text { T } & \text { T }\end{array}$ $\begin{array}{lllllll}0.4550222636579336 & 0.2816984637219779 & 0.5303949883509403 & \text { T } & \text { T }\end{array}$ $\begin{array}{llllll}0.75689140299999974 & 0.4965543409999995 & 0.0741721933333395 & F & F & F\end{array}$ $\begin{array}{llllll}0.7568914060000012 & 0.7568913409999993 & 0.0741721933333395 & F & F & F\end{array}$ $\begin{array}{llllll}0.4965543189999977 & 0.7568913409999993 & 0.0741721933333395 & F & F & F\end{array}$ $\begin{array}{lllllll}0.4965542930000026 & 0.2568913539999969 & 0.0741722013333330 & F & F & F\end{array}$ $\begin{array}{lllllll}0.2568913460000033 & 0.4965542950000028 & 0.0741722013333330 & F & F & F\end{array}$ $\begin{array}{lllllll}0.2568914200000023 & 0.2568913539999969 & 0.0741722013333330 & F & F & F\end{array}$ $\begin{array}{lllllll}0.9965543819999994 & 0.7568912950000026 & 0.0741722013333330 & F & F & F\end{array}$ $\begin{array}{lllllll}0.2568913890000033 & 0.9965543280000020 & 0.0741722013333330 & F & F & F\end{array}$ $\begin{array}{llllll}0.7568912999999995 & 0.2568913539999969 & 0.0741722013333330 & F & F & F\end{array}$ $\begin{array}{lllll}0.9965543329999988 & 0.2568913539999969 & 0.0741722013333330 & F & F\end{array}$ $\begin{array}{llllll}0.7568913490000000 & 0.9965543280000020 & 0.0741722013333330 & F & F & F\end{array}$ $\begin{array}{llllll}0.2568913890000033 & 0.7568912950000026 & 0.0741722013333330 & F & F & F\end{array}$ $\begin{array}{llllll}0.0034456799999987 & 0.0034456819999988 & 0.0758492213333355 & F & F & F\end{array}$ $\begin{array}{lllllll}0.5034457029999970 & 0.0034456619999972 & 0.0758492373333368 & F & F & F\end{array}$ $0.00344567399999820 .50344567900000210 .0758492373333368 \quad F \quad F \quad F$ $\begin{array}{llllll}0.5034457940000010 & 0.5034456790000021 & 0.0758492373333368 & F & F & F\end{array}$ $\begin{array}{lllllll}0.3367790249999985 & 0.3367790169999978 & 0.1500589058244657 & F & F & F\end{array}$ $\begin{array}{llllll}0.3367789719999976 & 0.8367790040000003 & 0.1500589218244670 & F & F & F\end{array}$ $\begin{array}{llllll}0.8367790920000004 & 0.8367790040000003 & 0.1500589218244670 & F & F & F\end{array}$ $0.83677899700000320 .33677899399999940 .1500589218244670 \quad F \quad F \quad F$ $\begin{array}{lllllll}0.8454804489765664 & 0.5824282795117171 & 0.1517299961083083 & F & F & F\end{array}$ $\begin{array}{lllllll}0.5824282575117152 & 0.5824282795117171 & 0.1517299961083083 & F & F & F\end{array}$ $\begin{array}{llllll}0.5824282385117172 & 0.8454805109765644 & 0.1517299961083083 & F & F & F\end{array}$ $0.08242828051172070 .58242823351172040 .1517300121083096 \quad F \quad F \quad F$ $\begin{array}{lllllll}0.3454805459765637 & 0.0824282865117141 & 0.1517300121083096 & F & F & F\end{array}$ $\begin{array}{lllllll}0.5824282745117202 & 0.0824282865117141 & 0.1517300121083096 & F & F & F\end{array}$ $\begin{array}{lllllll}0.0824283145117164 & 0.3454804769765616 & 0.1517300121083096 & F & F & F\end{array}$ $\begin{array}{llllll}0.3454805119765680 & 0.5824282335117204 & 0.1517300121083096 & F & F & F\end{array}$ $\begin{array}{lllllll}0.8454804259765680 & 0.0824282865117141 & 0.1517300121083096 & F & F & F\end{array}$ $0.08242832451171720 .84548041797656740 .1517300121083096 \quad F \quad F \quad F$ $\begin{array}{lllllll}0.0824282945117147 & 0.0824282865117141 & 0.1517300121083096 & F & F & F\end{array}$ $\begin{array}{lllllll}0.5824282345117169 & 0.3454804769765616 & 0.1517300121083096 & F & F & F\end{array}$ $\begin{array}{lllllll}0.9247598096318321 & 0.4248537578970074 & 0.2318770312745183 & \text { T } & \text { T } & \text { T }\end{array}$ $\begin{array}{llllll}0.4245300504399104 & 0.6605633178966013 & 0.2311011310209070 & \text { T } & \text { T }\end{array}$ $\begin{array}{llllll}0.6611617871875702 & 0.9247634616748307 & 0.2319148690809389 & \text { T } & \text { T }\end{array}$ $\begin{array}{lllllll}0.4247698110917345 & 0.9250973773516086 & 0.2309553606196963 & \text { T } & \text { T }\end{array}$ $\begin{array}{llllll}0.9250851664117445 & 0.6608335612727273 & 0.2319433202934455 & \text { T } & \text { T }\end{array}$ $\begin{array}{llllll}0.6597513466683889 & 0.4253652987371815 & 0.2318329654629707 & \text { T } & \text { T }\end{array}$ $\begin{array}{lllllll}0.9253784407696777 & 0.9248389823664099 & 0.2319943882225189 & \text { T } & \text { T }\end{array}$ $\begin{array}{lllllll}0.1610639331258898 & 0.4249065426717984 & 0.2310985880538158 & \text { T } & \text { T } & \text { T }\end{array}$ $\begin{array}{lllllll}0.1608682475767511 & 0.9243954904721655 & 0.2312015845237728 & \text { T } & \text { T }\end{array}$ $\begin{array}{lllllll}0.9245055868275230 & 0.1613396589859290 & 0.2320090293438611 & \text { T } & \text { T }\end{array}$ $\begin{array}{llllllll}0.4241364581460971 & 0.4245188133717217 & 0.2312750284453838 & \text { T } & \text { T }\end{array}$ $\begin{array}{lllllll}0.4250565589358417 & 0.1603676830393679 & 0.2309605837080410 & \text { T } & \text { T }\end{array}$ $\begin{array}{llllll}0.6694950767268434 & 0.6707087567614719 & 0.2353744583679979 & \text { T } & \text { T }\end{array}$ $\begin{array}{lllllll}0.6690587344395739 & 0.1707667260155266 & 0.2353909114956867 & \text { T } & \text { T }\end{array}$ $\begin{array}{lllllll}0.1702630500346816 & 0.1699954013401542 & 0.2353087053707386 & \text { T } & \text { T }\end{array}$ $\begin{array}{llllllll}0.1713063644908855 & 0.6691768897219640 & 0.2356495862893503 & \text { T } & \text { T }\end{array}$ $\begin{array}{lllllll}0.0038737345508064 & 0.0034262912198911 & 0.3054572825141874 & \text { T } & \text { T }\end{array}$ $\begin{array}{lllllll}0.0041663472809888 & 0.5030913951599366 & 0.3053200014028565 & \text { T } & \text { T }\end{array}$ 


$\begin{array}{lllllll}0.5026687567605909 & 0.0042684795079411 & 0.3052160371086151 & \mathrm{~T} & \mathrm{~T} & \mathrm{~T} \\ 0.5027908580278397 & 0.5036734934661933 & 0.3053887506144661 & \mathrm{~T} & \mathrm{~T} & \mathrm{~T} \\ 0.2559243476656242 & 0.7534068718036896 & 0.3070440461705592 & \mathrm{~T} & \mathrm{~T} & \mathrm{~T} \\ 0.7472852735102882 & 0.0128381627231961 & 0.3088972229765339 & \mathrm{~T} & \mathrm{~T} & \mathrm{~T} \\ 0.0148175350459051 & 0.2481330596488350 & 0.3091980354955269 & \mathrm{~T} & \mathrm{~T} & \mathrm{~T} \\ 0.7477485215185781 & 0.7504670387120972 & 0.3089676726794667 & \mathrm{~T} & \mathrm{~T} & \mathrm{~T} \\ 0.2562431335233079 & 0.5003880104787418 & 0.3071788803278907 & \mathrm{~T} & \mathrm{~T} & \mathrm{~T} \\ 0.2562912987596412 & 0.2539234915271214 & 0.3056524848829160 & \mathrm{~T} & \mathrm{~T} & \mathrm{~T} \\ 0.7473895960135981 & 0.5126675389194564 & 0.3088111725847613 & \mathrm{~T} & \mathrm{~T} & \mathrm{~T} \\ 0.4994763315339151 & 0.7554179773300032 & 0.3065782512688113 & \mathrm{~T} & \mathrm{~T} & \mathrm{~T} \\ 0.4994741599867017 & 0.2557693206947093 & 0.3068613031025282 & \mathrm{~T} & \mathrm{~T} & \mathrm{~T} \\ 0.0149789655869147 & 0.7477560801002620 & 0.3091262547112744 & \mathrm{~T} & \mathrm{~T} & \mathrm{~T} \\ 0.2560777084525214 & 0.0014502273697305 & 0.3064192300697913 & \mathrm{~T} & \mathrm{~T} & \mathrm{~T} \\ 0.7474811616254847 & 0.2504102483698291 & 0.3088563305654679 & \mathrm{~T} & \mathrm{~T} & \mathrm{~T} \\ 0.3330769676034663 & 0.8443207109142591 & 0.3944100594548806 & \mathrm{~T} & \mathrm{~T} & \mathrm{~T} \\ 0.3260545362630993 & 0.3239426762158649 & 0.3941004492563159 & \mathrm{~T} & \mathrm{~T} & \mathrm{~T} \\ 0.3244490107903704 & 0.5047544711142603 & 0.4434267353460133 & \mathrm{~T} & \mathrm{~T} & \mathrm{~T} \\ 0.4616193553448694 & 0.2586538055579943 & 0.4609559658362857 & \mathrm{~T} & \mathrm{~T} & \mathrm{~T} \\ 0.3295230517221758 & 0.3779170341930417 & 0.4411779749542633 & \mathrm{~T} & \mathrm{~T} & \mathrm{~T}\end{array}$

\section{Coordinates: $\mathrm{PO}_{\mathrm{x}}$ Structure without excess $\mathrm{O}$ (Fig. 3C)}

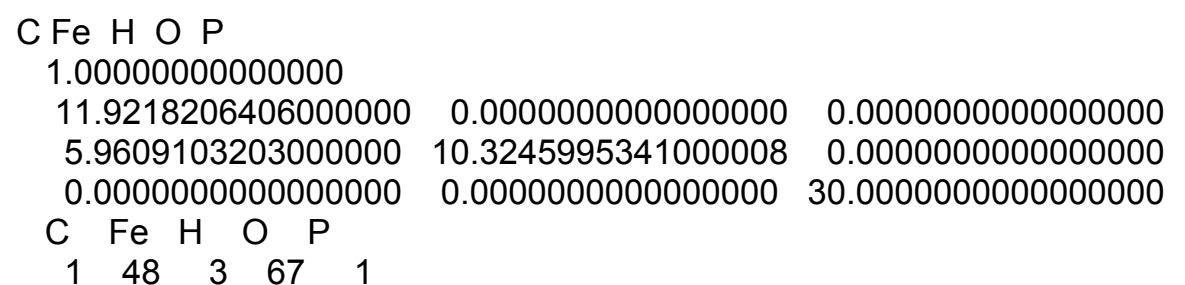

Selective dynamics

Direct

$\begin{array}{lllllll}0.2619575716023071 & 0.8227227547527147 & 0.4243186978232188 & \mathrm{~T} & \mathrm{~T} & \mathrm{~T} \\ 0.3367790249999985 & 0.3367790169999978 & 0.0327748283333378 & \mathrm{~F} & \mathrm{~F} & \mathrm{~F} \\ 0.3367789719999976 & 0.8367790040000003 & 0.0327748283333378 & \mathrm{~F} & \mathrm{~F} & \mathrm{~F} \\ 0.8367789850000023 & 0.3367790169999978 & 0.0327748283333378 & \mathrm{~F} & \mathrm{~F} & \mathrm{~F} \\ 0.8367790920000004 & 0.8367790040000003 & 0.0327748283333378 & \mathrm{~F} & \mathrm{~F} & \mathrm{~F} \\ 0.6701123229999979 & 0.6701123409999994 & 0.0530542513333359 & \mathrm{~F} & \mathrm{~F} & \mathrm{~F} \\ 0.1701123630000012 & 0.6701123409999994 & 0.0530542513333359 & \mathrm{~F} & \mathrm{~F} & \mathrm{~F} \\ 0.6701123019999997 & 0.1701123429999996 & 0.0530542513333359 & \mathrm{~F} & \mathrm{~F} & \mathrm{~F} \\ 0.1701123420000030 & 0.1701123429999996 & 0.0530542513333359 & \mathrm{~F} & \mathrm{~F} & \mathrm{~F} \\ 0.9198738180630173 & 0.1705895108739739 & 0.1120377980019782 & \mathrm{~F} & \mathrm{~F} & \mathrm{~F} \\ 0.1705895158739779 & 0.9198737280630169 & 0.1120377980019782 & \mathrm{~F} & \mathrm{~F} & \mathrm{~F} \\ 0.1705895288739754 & 0.4198737410630144 & 0.1120377980019782 & \mathrm{~F} & \mathrm{~F} & \mathrm{~F} \\ 0.4198737380630178 & 0.1705895108739739 & 0.1120377980019782 & \mathrm{~F} & \mathrm{~F} & \mathrm{~F} \\ 0.4198737570630158 & 0.4198737410630144 & 0.1120377980019782 & \mathrm{~F} & \mathrm{~F} & \mathrm{~F} \\ 0.9198737040630149 & 0.9198737280630169 & 0.1120377980019782 & \mathrm{~F} & \mathrm{~F} & \mathrm{~F} \\ 0.9198738370630153 & 0.4198737410630144 & 0.1120377980019782 & \mathrm{~F} & \mathrm{~F} & \mathrm{~F} \\ 0.4198737440630183 & 0.9198737280630169 & 0.1120377980019782 & \mathrm{~F} & \mathrm{~F} & \mathrm{~F} \\ 0.4198737190630197 & 0.6705895088739737 & 0.1120377980019782 & \mathrm{~F} & \mathrm{~F} & \mathrm{~F} \\ 0.9198737590630159 & 0.6705895088739737 & 0.1120377980019782 & \mathrm{~F} & \mathrm{~F} & \mathrm{~F} \\ 0.6705895158739779 & 0.9198737280630169 & 0.1120377980019782 & \mathrm{~F} & \mathrm{~F} & \mathrm{~F} \\ 0.6705894888739721 & 0.4198737410630144 & 0.1120377980019782 & \mathrm{~F} & \mathrm{~F} & \mathrm{~F} \\ 0.6693492149467335 & 0.6707578715564559 & 0.1719154579847516 & \mathrm{~T} & \mathrm{~T} & \mathrm{~T} \\ 0.1682635461309897 & 0.6710987623058040 & 0.1727219202100926 & \mathrm{~T} & \mathrm{~T} & \mathrm{~T} \\ 0.1705015020799177 & 0.1709552490296755 & 0.1740500482910640 & \mathrm{~T} & \mathrm{~T} & \mathrm{~T} \\ 0.6712061676882561 & 0.1694246038690609 & 0.1713408920543177 & \mathrm{~T} & \mathrm{~T} & \mathrm{~T}\end{array}$


$\begin{array}{llll}0.5006838321815849 & 0.0015770979995085 & 0.1937777634356339\end{array}$ $\begin{array}{llllll}0.5060125134260646 & 0.5023326292195364 & 0.1928721690384889 & \text { T } & \text { T }\end{array}$ $\begin{array}{llllll}0.0092104461446032 & 0.5007411428068137 & 0.1940663791364627 & \text { T } & \text { T }\end{array}$ $\begin{array}{lllllll}0.0021613575262281 & 0.0014815485127312 & 0.1939042380262788 & \text { T } & \text { T }\end{array}$ $\begin{array}{lllllll}0.3387944541367334 & 0.3359577773408404 & 0.2139597877205234 & \text { T } & \text { T }\end{array}$ $\begin{array}{lllllll}0.8390532332374997 & 0.3367320216137266 & 0.2138747344931524 & \text { T } & \text { T }\end{array}$ $\begin{array}{lllllll}0.3362801345970864 & 0.8358085253830843 & 0.2142923254537335 & \text { T } & \text { T }\end{array}$ $\begin{array}{lllllll}0.8371588189238504 & 0.8352842317996443 & 0.2147196660716703 & \text { T } & \text { T }\end{array}$ $\begin{array}{llllll}0.5897096746701481 & 0.0850634966481898 & 0.2755395325673334 & \text { T } & \text { T }\end{array}$ $\begin{array}{lllllll}0.0941131194350802 & 0.5865107152508895 & 0.2779680491302407 & \mathrm{~T} & \mathrm{~T}\end{array}$ $\begin{array}{lllllll}0.0892095368765942 & 0.3350036711750674 & 0.2795764535890550 & \text { T } & \text { T }\end{array}$ $\begin{array}{lllllll}0.5914333959529484 & 0.3265735579477196 & 0.2701085710003966 & \text { T } & \text { T }\end{array}$ $\begin{array}{lllllll}0.3319784416124426 & 0.5887735904483909 & 0.2754147198126748 & \text { T } & \text { T }\end{array}$ $\begin{array}{lllllll}0.3346501806347797 & 0.0885356540919197 & 0.2784889053279786 & \text { T } & \text { T }\end{array}$ $\begin{array}{lllllll}0.5899753508406324 & 0.5927057394076354 & 0.2726133553284106 & \text { T } & \text { T }\end{array}$ $\begin{array}{lllllll}0.0874504523796078 & 0.0883733391894070 & 0.2781444086253065 & \text { T } & \text { T }\end{array}$ $\begin{array}{lllllll}0.0930899655987584 & 0.8330904593996640 & 0.2779304996751906 & \text { T } & \text { T }\end{array}$ $\begin{array}{llllllll}0.5874382749586672 & 0.8350733529871176 & 0.2775232086292889 & \mathrm{~T} & \mathrm{~T}\end{array}$ $\begin{array}{lllllll}0.8386498275549391 & 0.5867066688622847 & 0.2764337785438863 & \text { T } & \text { T }\end{array}$ $\begin{array}{lllllll}0.8361041715993590 & 0.0849256199731952 & 0.2761534348805053 & \text { T } & \text { T }\end{array}$ $\begin{array}{lllllll}0.8386562545191660 & 0.8357692260031563 & 0.3190562457606586 & \text { T } & \text { T }\end{array}$ $\begin{array}{lllllll}0.3399767760119701 & 0.8354855462144603 & 0.3329382213062622 & \text { T } & \text { T }\end{array}$ $\begin{array}{lllllll}0.8289682161118862 & 0.3402201584788749 & 0.3296649960037770 & \text { T } & \text { T }\end{array}$ $\begin{array}{lllllll}0.3450069066222648 & 0.3356244437705516 & 0.3312590601699180 & \text { T } & \text { T }\end{array}$ $\begin{array}{lllllll}0.3106401628142970 & 0.7535837695495650 & 0.4518615560340443 & \text { T } & \text { T }\end{array}$ $\begin{array}{lllllll}0.2109182691236538 & 0.9213953699272870 & 0.4386263465580300 & \text { T } & \text { T }\end{array}$ $\begin{array}{lllllll}0.1889368460564779 & 0.8022581414600138 & 0.4096513853919617 & \text { T } & \text { T }\end{array}$ $\begin{array}{lllllll}0.7568914029999974 & 0.4965543409999995 & 0.0741721933333395 & F & F & F\end{array}$ $\begin{array}{lllllll}0.7568914060000012 & 0.7568913409999993 & 0.0741721933333395 & F & F & F\end{array}$ $\begin{array}{llllllllll}0.4965543189999977 & 0.7568913409999993 & 0.0741721933333395 & F & F & F\end{array}$ $\begin{array}{llllllll}0.2568914200000023 & 0.2568913539999969 & 0.0741722013333330 & F & F & F\end{array}$ $\begin{array}{llllllll}0.2568913890000033 & 0.9965543280000020 & 0.0741722013333330 & F & F & F\end{array}$ $\begin{array}{lllllllll}0.7568912999999995 & 0.2568913539999969 & 0.0741722013333330 & F & F & F\end{array}$ $\begin{array}{llllllllll}0.9965543329999988 & 0.2568913539999969 & 0.0741722013333330 & F & F & F\end{array}$ $\begin{array}{llllllll}0.7568913490000000 & 0.9965543280000020 & 0.0741722013333330 \quad F & F & F\end{array}$ $\begin{array}{lllllll}0.2568913890000033 & 0.7568912950000026 & 0.0741722013333330 & F & F & F\end{array}$ $\begin{array}{lllllllllll}0.2568913460000033 & 0.4965542950000028 & 0.0741722013333330 & F & F & F\end{array}$ $\begin{array}{lllllllllll}0.4965542930000026 & 0.2568913539999969 & 0.0741722013333330 & F & F & F\end{array}$ $\begin{array}{llllllll}0.9965543819999994 & 0.7568912950000026 & 0.0741722013333330 & F & F & F\end{array}$ $\begin{array}{lllllll}0.0034456799999987 & 0.0034456819999988 & 0.0758492213333355 & F & F & F\end{array}$ $0.00344567399999820 .50344567900000210 .0758492373333368 \quad F \quad F \quad F$ $\begin{array}{llllllllll}0.5034457029999970 & 0.0034456619999972 & 0.0758492373333368 & F & F & F\end{array}$ $\begin{array}{llllllll}0.5034457940000010 & 0.5034456790000021 & 0.0758492373333368 & F & F & F\end{array}$ $\begin{array}{llllllll}0.3367790249999985 & 0.3367790169999978 & 0.1500589058244657 & F & F & F\end{array}$ $0.83677909200000040 .83677900400000030 .1500589218244670 \quad F \quad F \quad F$ $\begin{array}{llllllllll}0.8367789970000032 & 0.3367789939999994 & 0.1500589218244670 & F & F & F\end{array}$ $\begin{array}{llllllll}0.3367789719999976 & 0.8367790040000003 & 0.1500589218244670 & F & F & F\end{array}$ $0.84548044897656640 .58242827951171710 .1517299961083083 \quad F \quad F \quad F$ $\begin{array}{lllllll}0.5824282575117152 & 0.5824282795117171 & 0.1517299961083083 & F & F & F\end{array}$ $\begin{array}{lllllllll}0.5824282385117172 & 0.8454805109765644 & 0.1517299961083083 & F & F & F\end{array}$ $\begin{array}{lllllllll}0.8454804259765680 & 0.0824282865117141 & 0.1517300121083096 & F & F & F\end{array}$ $\begin{array}{lllllll}0.0824283245117172 & 0.8454804179765674 & 0.1517300121083096 & F & F & F\end{array}$ $\begin{array}{lllllllll}0.0824282805117207 & 0.5824282335117204 & 0.1517300121083096 & F & F & F\end{array}$ $\begin{array}{llllllllll}0.5824282345117169 & 0.3454804769765616 & 0.1517300121083096 & F & F & F\end{array}$ $\begin{array}{lllllllllll}0.3454805459765637 & 0.0824282865117141 & 0.1517300121083096 & F & F & F\end{array}$ $\begin{array}{llllllll}0.5824282745117202 & 0.0824282865117141 & 0.1517300121083096 & F & F & F\end{array}$ $\begin{array}{lllllllll}0.3454805119765680 & 0.5824282335117204 & 0.1517300121083096 & F & F & F\end{array}$ $\begin{array}{llllllllll}0.0824283145117164 & 0.3454804769765616 & 0.1517300121083096 & F & F & F\end{array}$ $0.08242829451171470 .08242828651171410 .1517300121083096 \quad F \quad F \quad F$ $\begin{array}{lllllll}0.1606421828651814 & 0.9265703491515835 & 0.2318058108333076 & \text { T } & \text { T } & \text { T }\end{array}$ $\begin{array}{lllllll}0.1636740076965498 & 0.4225162694398120 & 0.2327398676837805 & \mathrm{~T}\end{array} \mathrm{~T}$ 


\begin{tabular}{|c|c|c|c|}
\hline 0.9275539341095604 & 0.4233201574961208 & 0.2323626785991314 & \\
\hline 0.4308383194942275 & 0.6587016435111787 & 0.2318801320991976 & \\
\hline 0.6601325559761031 & 0.9224144658511276 & 0.2320542805638595 & $\mathrm{~T}$ \\
\hline 0.9268802317211479 & 0.1611318263103339 & 0.2317375588177012 & $\mathrm{~T}$ \\
\hline 0.4232285586188979 & 0.4278187870334833 & 0.2319125619258495 & $\mathrm{~T}$ \\
\hline 0.4235747828847665 & 0.1599204031003992 & 0.2315226378305857 & \\
\hline 0.4229056698073192 & 0.9248905217661200 & 0.2319749784051536 & $\mathrm{~T}$ \\
\hline 0.9253131398974215 & 0.9236616415183619 & 0.2322265400410046 & $T$ \\
\hline 0.6612980955263049 & 0.4288809280738783 & 0.2316275538097600 & \\
\hline 0.9245949057917323 & 0.6588642066014134 & 0.2322376477632657 & \\
\hline 0.1691859007838965 & 0.1731960062844584 & 0.2374387959963315 & \\
\hline 0.6714523271973930 & 0.6700327959976822 & 0.2345052653699922 & \\
\hline 0.1637670499843879 & 0.6731610179521929 & 0.2344905572397381 & $\mathrm{~T}$ \\
\hline 0.6767098635814424 & 0.1577070152952434 & 0.2328608499839 & \\
\hline 0.0033966909076883 & 0.0042461315969671 & 0.3056980232886625 & $\mathrm{~T}$ \\
\hline 0.0048683853060026 & 0.5033706475617138 & 0.3062750110836595 & $\mathrm{~T}$ \\
\hline 0.5037609134115323 & 0.0031930006641863 & 0.3057350725396026 & $\mathrm{~T}$ \\
\hline 0.5115817739453465 & 0.5007202848613390 & 0.3182407807555421 & \\
\hline 0.7553646660792305 & 0.2526590504158212 & 0.3046550738418300 & $\mathrm{~T}$ \\
\hline 0.2527399623641298 & 0.0053484707743983 & 0.3083393437325372 & \\
\hline 0.0163374853515689 & 0.7475242288332340 & 0.3098069074466078 & T \\
\hline 0.2563399695035917 & 0.7551595215058005 & 0.3068748079344006 & \\
\hline 0.7484268446631633 & 0.0137828714030370 & 0.3092081060579734 & $T$ \\
\hline-0.0001516168305866 & 0.2564856341033077 & 0.3107483849565120 & $\mathrm{~T}$ \\
\hline 0.7497763422671649 & 0.7469032112435223 & 0.3086972214051836 & \\
\hline 0.2538698448733802 & 0.4983087989941297 & 0.3097852649613120 & $\mathrm{~T}$ \\
\hline 0.7527726716360067 & 0.5029460193825011 & 0.3054812687396005 & \\
\hline 0.5054010671265929 & 0.7493560580280852 & 0.3058991259578122 & \\
\hline 0.5020912980490221 & 0.2502897734215627 & 0.3040993949953253 & \\
\hline 0.2556198456436373 & 0.2538831622198657 & 0.3113017367524435 & T \\
\hline 0.6795746359857417 & 0.3909506942350487 & 0.3813219863995820 & \\
\hline 0.4602271771242842 & 0.3861639034653250 & 0.3813981220226104 & \\
\hline 0.3550598579834439 & 0.8106979156788974 & & \\
\hline 0.5541561251771115 & 0.4183263991191635 & 0.3607588197368374 & \\
\hline
\end{tabular}

\section{Coordinates: $\mathrm{PO}_{\mathrm{x}}$ Structure stabilized with excess $\mathrm{O}$ (Fig. 3D)}

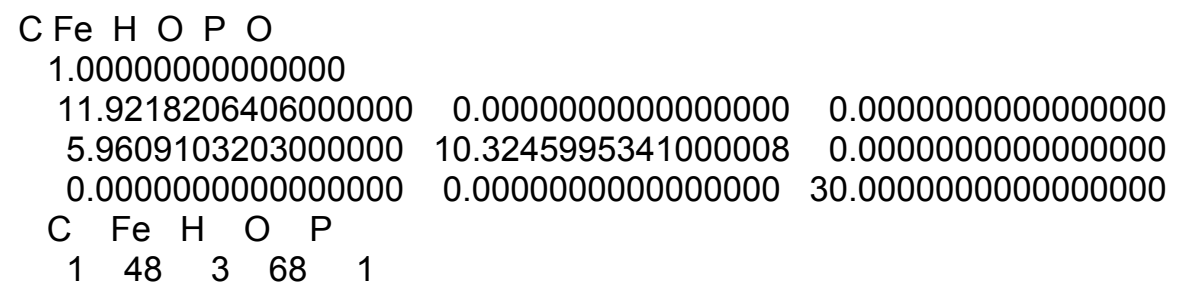

Selective dynamics

Direct

$\begin{array}{lllllll}0.2426997367254358 & 0.8430199625004835 & 0.4303168258267705 & \text { T } & \text { T }\end{array}$ $\begin{array}{lllllll}0.3367790249999985 & 0.3367790169999978 & 0.0327748283333378 & F & F & F\end{array}$ $\begin{array}{llllllll}0.3367789719999976 & 0.8367790040000003 & 0.0327748283333378 & F & F & F\end{array}$ $\begin{array}{llllllll}0.8367789850000023 & 0.3367790169999978 & 0.0327748283333378 & F & F & F\end{array}$ $0.83677909200000040 .83677900400000030 .0327748283333378 \quad F \quad F \quad F$ $0.67011232299999790 .67011234099999940 .0530542513333359 \quad F \quad F \quad F$ $0.17011236300000120 .67011234099999940 .0530542513333359 \quad F \quad F \quad F$ $\begin{array}{lllllll}0.6701123019999997 & 0.1701123429999996 & 0.0530542513333359 & F & F & F\end{array}$ $\begin{array}{lllllll}0.1701123420000030 & 0.1701123429999996 & 0.0530542513333359 & F & F & F\end{array}$ $\begin{array}{llllllll}0.9198738180630173 & 0.1705895108739739 & 0.1120377980019782 & F & F & F\end{array}$ $\begin{array}{llllllll}0.1705895158739779 & 0.9198737280630169 & 0.1120377980019782 & F & F & F\end{array}$ 0.17058952887397540 .41987374106301440 .1120377980019782 F $F \quad F$ $0.41987373806301780 .17058951087397390 .1120377980019782 \quad F \quad F \quad F$ $0.41987375706301580 .41987374106301440 .1120377980019782 \quad F \quad F \quad F$ 0.91987370406301490 .91987372806301690 .1120377980019782 F F F 
$\begin{array}{llll}0.9198738370630153 & 0.4198737410630144 & 0.1120377980019782\end{array}$ $\begin{array}{llll}0.4198737440630183 & 0.9198737280630169 & 0.1120377980019782\end{array}$ $\begin{array}{lllll}0.4198737190630197 & 0.6705895088739737 & 0.1120377980019782\end{array}$ $\begin{array}{llll}0.9198737590630159 & 0.6705895088739737 & 0.1120377980019782\end{array}$ $\begin{array}{llll}0.6705895158739779 & 0.9198737280630169 & 0.1120377980019782\end{array}$ $\begin{array}{llll}0.6705894888739721 & 0.4198737410630144 & 0.1120377980019782\end{array}$ $\begin{array}{llll}0.6707772623666959 & 0.6709969562090630 & 0.1719098080902339\end{array}$ 0.17005360433545590 .66949008018003450 .1717754532135548 $\begin{array}{llll}0.1707566587008672 & 0.1690674241044526 & 0.1757320488964426\end{array}$ $\begin{array}{llll}0.6708947537853311 & 0.1697281360024679 & 0.1705881823201511\end{array}$ $\begin{array}{llll}0.4990942117099981 & 0.0015390767316343 & 0.1940369969197988\end{array}$ $\begin{array}{llll}0.5010998264101963 & 0.5019895081298559 & 0.1917325160798927\end{array}$ $\begin{array}{llll}0.0034583854729740 & 0.5034776589152488 & 0.1942721683027426\end{array}$ $\begin{array}{lllll}0.0037985172061815 & 0.0076130414102817 & 0.1941985069692318\end{array}$ $\begin{array}{llll}0.3350662445961169 & 0.3349004360900867 & 0.2143250216587707\end{array}$ $\begin{array}{llll}0.8374868195272629 & 0.3374905658209363 & 0.2143636243727780\end{array}$ $\begin{array}{llll}0.3351855665605427 & 0.8375030617314730 & 0.2147546229921995\end{array}$ $\begin{array}{llll}0.8360297878164923 & 0.8378042301871383 & 0.2145192253702264\end{array}$ $\begin{array}{llll}0.5878283008108364 & 0.0838341787986145 & 0.2749708574602026\end{array}$ $\begin{array}{llll}0.0875475141693236 & 0.5869840317128773 & 0.2764504516793453\end{array}$ $\begin{array}{llll}0.0880350874925066 & 0.3339399915725514 & 0.2830527483814114\end{array}$ $\begin{array}{llll}0.5921503411330680 & 0.3265183782781626 & 0.2694693375923842\end{array}$ $0.32866840602882190 .5931742095561894 \quad 0.2730668704484231$ $\begin{array}{llll}0.3320019931285025 & 0.0910333949679985 & 0.2821051106220260\end{array}$ $\begin{array}{lllll}0.5863808253358364 & 0.5943259469444687 & 0.2730170247219950\end{array}$ $\begin{array}{llll}0.0887075619308784 & 0.0899669282936022 & 0.2825648744586045\end{array}$ $\begin{array}{llll}0.0897934378291780 & 0.8361959241716339 & 0.2772478565927898\end{array}$ $\begin{array}{llll}0.5830320566633878 & 0.8357447545673630 & 0.2781052706930630\end{array}$ $\begin{array}{llll}0.8348998778972259 & 0.5870450697177054 & 0.2763474574735902\end{array}$ $\begin{array}{llll}0.8377243044525879 & 0.0837220639932401 & 0.2755532604706715\end{array}$ 0.83696757460006590 .83590565360903910 .3188298369001125 $\begin{array}{llll}0.3437157733408960 & 0.8164645776647291 & 0.3406191093221615\end{array}$ $\begin{array}{llll}0.8105553992219150 & 0.3472477735256050 & 0.3326617087828899\end{array}$ $\begin{array}{llll}0.3530714767194442 & 0.3467645042201252 & 0.3327393710946205\end{array}$ $\begin{array}{llll}0.3305930177519573 & 0.7653798596378094 & 0.4463529695762322\end{array}$ $\begin{array}{llll}0.1889298631466504 & 0.9214474704523214 & 0.4546336654960555\end{array}$ 0.18130440444426950 .80197002852732220 .4204326857574606 $\begin{array}{llll}0.7568914029999974 & 0.4965543409999995 & 0.0741721933333395\end{array}$ $0.75689140600000120 .7568913409999993 \quad 0.0741721933333395$ $\begin{array}{llll}0.4965543189999977 & 0.7568913409999993 & 0.0741721933333395\end{array}$ $0.2568914200000023 \quad 0.25689135399999690 .0741722013333330$ $0.25689138900000330 .9965543280000020 \quad 0.0741722013333330$ $0.7568912999999995 \quad 0.25689135399999690 .0741722013333330$ $\begin{array}{llll}0.9965543329999988 & 0.2568913539999969 & 0.0741722013333330\end{array}$ $\begin{array}{llll}0.7568913490000000 & 0.9965543280000020 & 0.0741722013333330\end{array}$ $0.25689138900000330 .7568912950000026 \quad 0.0741722013333330$ 0.25689134600000330 .49655429500000280 .0741722013333330 $\begin{array}{llll}0.4965542930000026 & 0.2568913539999969 & 0.0741722013333330\end{array}$ $\begin{array}{llll}0.9965543819999994 & 0.7568912950000026 & 0.0741722013333330\end{array}$ $\begin{array}{llll}0.0034456799999987 & 0.0034456819999988 & 0.0758492213333355\end{array}$ 0.00344567399999820 .50344567900000210 .0758492373333368 $\begin{array}{llll}0.5034457029999970 & 0.0034456619999972 & 0.0758492373333368\end{array}$ $\begin{array}{lllll}0.5034457940000010 & 0.5034456790000021 & 0.0758492373333368\end{array}$ $\begin{array}{llll}0.3367790249999985 & 0.3367790169999978 & 0.1500589058244657\end{array}$ $\begin{array}{llll}0.8367790920000004 & 0.8367790040000003 & 0.1500589218244670\end{array}$ $\begin{array}{lllll}0.8367789970000032 & 0.3367789939999994 & 0.1500589218244670\end{array}$ $\begin{array}{llll}0.3367789719999976 & 0.8367790040000003 & 0.1500589218244670\end{array}$ $\begin{array}{lllll}0.8454804489765664 & 0.5824282795117171 & 0.1517299961083083\end{array}$ $\begin{array}{llll}0.5824282575117152 & 0.5824282795117171 & 0.1517299961083083\end{array}$ $\begin{array}{lllll}0.5824282385117172 & 0.8454805109765644 & 0.1517299961083083\end{array}$ $\begin{array}{llll}0.8454804259765680 & 0.0824282865117141 & 0.1517300121083096\end{array}$

$F \quad F \quad F$ $F F$ $F \quad F$ $F \quad F \quad F$ $F F$ $F F F$ $T \quad T \quad T$ $T \quad T \quad T$ $T T T$ $T T T$ $T \quad T \quad T$ $T \quad T \quad T$ $T T T$ $\begin{array}{ccc}T & T\end{array}$ $T \quad T \quad T$ $T T T$ $T T T$ $T T T$ $T T T$ $T T T$ $T T T$ $T T$ $T T T$ $T T T$ $T T T$ $T T T$ $T T T$ $T T T$ $T T T$ $T \quad T \quad T$ $\mathrm{T} T \mathrm{~T}$ $T T T$ $T T T$ $T T$ $T T T$ $T T T$ $T T T$ $F \quad F$ $F F F$ $F F$ $F F$ $F F F$ $F F$ $F F F$ $F F F$ $F F F$ $F F F$ $F F F$ $F F$ $F F$ $F F F$ $F \quad F$ $F F F$ $F F F$ $F F F$ $F F$ $F F F$ $F F F$ $F F F$ $F F$ $F F$ 


\begin{tabular}{|c|c|c|c|}
\hline & & & \\
\hline & & & \\
\hline 5824282345117169 & 0.3454804769765616 & 0.1517300121083096 & $\mathrm{~F}$ \\
\hline 0.3454805459765637 & 0.0824282865117141 & 0.1517300121083096 & $\mathrm{~F}$ \\
\hline 0.5824282745117202 & 0.0824282865117141 & 0.1517300121083096 & \\
\hline 3454805119765680 & 0.5824282335117204 & 0.1517300121083096 & \\
\hline 0824283145117164 & 0.3454804769765616 & 0.1517300121083096 & $\mathrm{~F}$ \\
\hline 0.0824282945117147 & 0.0824282865117141 & 0.1517300121083096 & \\
\hline 0.1602634394525633 & 0.9251212685622947 & 0.2319245590842856 & \\
\hline 00525563067735 & 0.4235209843463648 & 0.2323027248191135 & $\mathrm{~T}$ \\
\hline 0.9255577469851450 & 0.4245212990890444 & 0.2322390269200228 & $\mathrm{~T}$ \\
\hline 0.4255775666035889 & 0.6581423316789886 & 0.2316345913831395 & $\mathrm{~T}$ \\
\hline 0.6593365772238431 & 0.9249157796999975 & 0.2317536569251348 & $\mathrm{~T}$ \\
\hline 0.9267469445512453 & 0.1620249270516422 & 0.2316227458006051 & $\mathrm{~T}$ \\
\hline 0.4246617471688680 & 0.4248132506023413 & 0.2303079870136444 & $\mathrm{~T}$ \\
\hline 0.4221180561689920 & 0.1595885019780769 & 0.2316091772673404 & $\mathrm{~T}$ \\
\hline 0.4236626059904972 & 0.9239530590926860 & 0.2319231392458298 & $\mathrm{~T}$ \\
\hline 0.9259665552648944 & 0.9247568356077042 & 0.2317958789679369 & $\mathrm{~T}$ \\
\hline 0.6581191132523875 & 0.4263160092600391 & 0.2303672691172322 & $\mathrm{~T}$ \\
\hline 0.9246440036816105 & 0.6607955829657535 & 0.2319259267800531 & $\mathrm{~T}$ \\
\hline 0.167743193799837 & 0.1744710062275246 & 0.2396885877675440 & $\mathrm{~T}$ \\
\hline 0.6686479553490010 & 0.6714234768590004 & 0.2342898357245237 & $\mathrm{~T}$ \\
\hline 0.1695671733840851 & 0.6705103670005140 & 0.2341356985135649 & $\mathrm{~T}$ \\
\hline 0.6723192212263764 & 0.1657531087801697 & 0.2326572701009601 & $\mathrm{~T}$ \\
\hline 0.0020375530389523 & 0.0037156085597254 & 0.3062985330118104 & $\mathrm{~T}$ \\
\hline 0.0016988356486044 & 0.5060631004781149 & 0.3064902080373377 & T \\
\hline 0.5043195944752942 & 0.0038456979200639 & 0.3064240842636421 & $\mathrm{~T}$ \\
\hline 0.5070641925874392 & 0.4956666021235638 & 0.3062637740060170 & $\mathrm{~T}$ \\
\hline 0.7529956505114457 & 0.2534115809467733 & 0.3011453557951511 & $\mathrm{~T}$ \\
\hline 0.2579860322962136 & -0.0033771534051150 & 0.3080811448112375 & $\mathrm{~T}$ \\
\hline 0.01451680106 & 0.7476795395563530 & 178211076062 & $\mathrm{~T}$ \\
\hline 0.2560171434164828 & 0.7500540586702303 & 0.3055673281245562 & \\
\hline 0.7479399575688169 & 0.0133498716324851 & 0.3075594054207559 & $\mathrm{~T}$ \\
\hline-0.0095277983232033 & 0.2610519640577347 & 0.3130285141429557 & $\mathrm{~T}$ \\
\hline 0.7477600586933458 & 0.7476068205415135 & 0.3081492851246961 & $\mathrm{~T}$ \\
\hline 0.2560814078069015 & 0.5026512543904336 & 0.3038496991695445 & $\mathrm{~T}$ \\
\hline 0.7503425883762522 & 0.5031007626039117 & 0.3036242849342405 & $\mathrm{~T}$ \\
\hline 0.5015646390599244 & 0.7509774024561957 & 0.3061664248720363 & \\
\hline 0.5039549343570701 & 0.2533985528552692 & 0.3010337510692644 & \\
\hline 0.2586606143355886 & 0.2612635883347319 & 0.3129444767093515 & $\mathrm{~T}$ \\
\hline 0.6578471501987977 & 0.4118711273278006 & 0.3733861932880380 & \\
\hline 0.4402635412886087 & 0.4126586816329327 & 0.3734474253903807 & \\
\hline 0.2746268155866990 & 0.8942547692060715 & 0.3932745242618966 & \\
\hline 0.4416883729723122 & 0.6286911209879013 & 0.3763742806521956 & $\mathrm{~T}$ \\
\hline & & & \\
\hline
\end{tabular}




\section{References}

1. Lennie, A. R.; Condon, N. G.; Leibsle, F. M.; Murray, P. W.; Thornton, G.; Vaughan, D. J., Structures of $\mathrm{Fe}_{3} \mathrm{O}_{4}(111)$ Surfaces Observed by Scanning Tunneling Microscopy. Phys. Rev. B 1996, 53, 10244-10253.

2. Parkinson, G. S., Iron Oxide Surfaces. Surf. Sci. Rep. 2016, 71, 272-365.

3. Walenta, C. A.; Crampton, A. S.; Xu, F.; Heiz, U.; Friend, C. M., Chemistry of Methanol and Ethanol on Ozone-Prepared $\alpha-\mathrm{Fe}_{2} \mathrm{O}_{3}(0001)$. J. Phys. Chem. C 2018, 122, 25404-25410.

4. Harrison, A. G.; Jones, E. G.; Gupta, S. K.; Nagy, G. P., Total Cross Sections for Ionization by Electron Impact. Can. J. Chem. 1966, 44, 1967-1973.

5. Gupta, D.; Antony, B., Electron Impact Ionization of Cycloalkanes, Aldehydes, and Ketones. J. Chem. Phys. 2014, 141, 054303.

6. Bull, J. N.; Harland, P. W.; Vallance, C., Absolute Total Electron Impact Ionization Cross-Sections for Many-Atom Organic and Halocarbon Species. J. Phys. Chem. A 2012, 116, 767-777.

7. Nist Chemistry Webbook, Nist Standard Reference Database Number 69. Mallard, P. J. L. a. W. G., Ed. National Institute of Standards and Technology, Gaithersburg MD, 20899. (accessed June 5, 2018).

8. Henderson, M. A.; Jin, T.; White, J. M., A TPD/AES Study of the Interaction of Dimethyl Methylphosphonate with Iron Oxide $\left(a-\mathrm{Fe}_{2} \mathrm{O}_{3}\right)$ and Silicon Dioxide. J. Phys. Chem. 1986, 90, 4607-4611.

9. Herman, G. S.; McDaniel, E. P.; Joyce, S. A., Interaction of $\mathrm{D}_{2} \mathrm{O}$ with the $\mathrm{Fe}_{3} \mathrm{O}_{4}(111)$ and the Biphase Ordered Structures on $\alpha-\mathrm{Fe}_{2} \mathrm{O}_{3}(0001)$. J. Electron. Spectrosc. Relat. Phenom. 1999, 101-103, 433-438.

10. Sala, A.; Marchetto, H.; Qin, Z. H.; Shaikhutdinov, S.; Schmidt, T.; Freund, H. J., Defects and Inhomogeneities in $\mathrm{Fe}_{3} \mathrm{O}_{4}(111)$ Thin Film Growth on Pt(111). Phys. Rev. B 2012, 86, 155430.

11. Head, A. R.; Tsyshevsky, R.; Trotochaud, L.; Eichhorn, B.; Kuklja, M. M.; Bluhm, H., Electron Spectroscopy and Computational Studies of Dimethyl Methylphosphonate. J. Phys. Chem. A 2016, 120, 1985-1991.

12. Head, A. R., et al., Thermal Desorption of Dimethyl Methylphosphonate from $\mathrm{MoO}_{3}$. Catal. Struct. React. 2017, 3, 112-118.

13. Tang, X.; Hicks, Z.; Wang, L.; Ganteför, G.; Bowen, K. H.; Tsyshevsky, R.; Sun, J.; Kuklja, M. M., Adsorption and Decomposition of Dimethyl Methylphosphonate on Size-Selected $\left(\mathrm{MoO}_{3}\right)_{3}$ Clusters. Phys. Chem. Chem. Phys. 2018, 20, 4840-4850.

14. Trotochaud, L.; Head, A. R.; Büchner, C.; Yu, Y.; Karslıoğlu, O.; Tsyshevsky, R.; Holdren, S.; Eichhorn, B.; Kuklja, M. M.; Bluhm, H., Room Temperature Decomposition of Dimethyl Methylphosphonate on Cuprous Oxide Yields Atomic Phosphorus. Surf. Sci. 2019, 680, 75-87.

15. Bomben, J. F. M. W. F. S. P. E. S. K. D., Handbook of X-Ray Photoelectron Spectroscopy; Perkin Elmer Corporation Physical Electronics Division: 6509 Flying Cloud Drive, Eden Prairie, Minnesota 55344, United States of America, 1992. 